\title{
Spin versus Boson Baths in Nonlinear Spectroscopy
}

\author{
Y. Zhao, ${ }^{\dagger}$ V. Chernyak, ${ }^{\dagger}$ and S. Mukamel ${ }^{*, \hbar}$ \\ Department of Chemistry and Rochester Theory Center for Optical Science and Engineering, \\ University of Rochester, Rochester, New York 14627
}

Received: April 8, 1998; In Final Form: May 26, 1998

\begin{abstract}
The third-order optical response function of a chromophore coupled to a bath consisting of noninteracting two-level systems (TLSs) undergoing stochastic jumps is calculated. The results can be applied to a broad class of nonlinear optical techniques in single-molecule spectroscopy and bulk measurements in glasses, polymers, and mixed crystals. In the limit of many TLSs weakly coupled to the chromophore, the response can be obtained using the second-order cumulant expansion and resembles that of the Brownian oscillator model. Quantum corrections to the spectral density which accounts for the Stokes shift can be included in this limit using the fluctuation-dissipation theorem. However, the temperature dependence predicted by the two models is very different. Corrections to the cumulant expansion to fourth order in coupling strength are derived.
\end{abstract}

\section{Introduction}

Nonlinear spectroscopies in the condensed phase provide a direct probe for the coupling of chromophores to the surrounding medium (solvent, glass, host crystal, protein, etc.). ${ }^{1-3}$ In many situations, e.g., pentacene molecules in $p$-terphenyl crystals, the local environment of the chromophore can be modeled as a collection of flipping two-level systems (TLSs) which modulate its transition frequency. Such spectral jumps have been observed by repeated fast scanning of the zero-phonon line $e^{4-10}$ in the newly developed single-molecule spectroscopy (SMS). The TLS model has been quite successful in explaining anomalous specific heat and thermal conductivity of amorphous materials. ${ }^{12-19}$ The excess specific heat of glasses (in addition to the Debye specific heat) varies linearly with temperature in contrast to the $\sim T^{3}$ contributions from phonons at low temperatures. This suggests the existence of additional degrees of freedom in glasses with a roughly constant density of states. Relaxation by TLS tunneling at low temperature can be attributed to coupling of the TLS to acoustic phonons ${ }^{20-23}$ in analogy with phonon-assisted intermolecular transfer. ${ }^{24,25}$ For higher temperatures, however, introduction of soft phonon modes with a crossover to anharmonicity at low frequencies seems necessary. ${ }^{26-30}$

Theories of TLS frequency modulation were first developed by Anderson and Weiss ${ }^{31,32}$ for electron paramagnetic resonance and later extended by Kubo. ${ }^{33-35}$ Interest in the TLS model has spanned well over half a century. Line-shape functions and photon-echo signals have been calculated by many authors ${ }^{36-44}$ and compared with traditional measurements of bulk materials. Interactions between TLS have been incorporated as well. ${ }^{45}$ Studies of single molecules in solids came as a recent development of optical spectroscopy which combines near-field microscopy and high-resolution lasers. ${ }^{4-6,10,11,46-48}$ In contrast to traditional measurements, SMS reveals a distribution of the dynamic fluctuations of the host TLS instead of merely measuring an ensemble average. Much more information is

\footnotetext{
Department of Chemistry.

$\doteqdot$ Rochester Theory Center for Optical Science and Engineering.
}

contained in a distribution compared to its average or moments. SMS therefore calls for a more detailed microscopic theory to provide a precise description of the host dynamic distribution. Application of SMS has been found mostly in line-shape measurements. ${ }^{7,8,49-51}$ For example, line-width distributions of guest molecules embedded in a solid matrix (such as terrylene in polyethylene) have been measured at low temperatures. Nonlinear optical measurements, which are usually performed in bulk solids, are adopted for SMS.

In this paper we generalize the earlier treatments of the TLS bath and derive closed expressions for the complete third-order response function. These expressions are not limited to the photon echo ${ }^{38,40}$ and may be used for a broad range of measurements (e.g., pump-probe, hole-burning, and four-wave mixing). In the limit of many TLSs weakly coupled to the chromophore, the response functions can be calculated using the second-order cumulant expansion, resulting in an expression resembling the Brownian oscillator model. In this case we can further use the fluctuation-dissipation theorem to predict the full temperature dependence of the response including the timedependent Stokes shift (which is neglected in stochastic models). The Brownian oscillator model, which has been successfully utilized in modeling the condensed-phase measurements at room temperature ${ }^{3}$ predicts a different temperature dependence of the response (the real part of the spectral density function is dominant at high temperatures, while the temperature-independent imaginary part becomes significant at low temperatures). Photon-echo studies of a dye molecule (IR144) in a polymer glass (PMMA) show that many features of the host-guest system have been successfully described by the oscillator bath and its temperature-dependent response functions. ${ }^{52}$ For liquid ethylene glycol solvent, however, harmonic bath models capture the temperature dependence of the spectral density only in the high-frequency regime. ${ }^{53}$ Temperature dependence different from the boson bath has also been reported in organic polymers PMMA and PVA in the temperature range $30-300 \mathrm{~K}^{54}$ The temperature dependence of the TLS and Brownian oscillator model will be compared. 
In Section II, we describe the Brownian oscillator model and its optical response functions. ${ }^{3,55}$ The spectral density is discussed, and the third-order response is related to spectral broadening functions via the second-order cumulant expansion. In Section III, we derive the complete third-order response function for the TLS model which generalizes earlier calculations restricted to the three-pulse echo. In Section IV, we discuss the general scenario of a chromophore weakly coupled to a bath of many surrounding TLSs using the cumulant expansion. We extend the TLS model to include the imaginary part of the spectral density function by making use of the fluctuation-dissipation theorem. Absorption line-shape functions are calculated and compared with the Brownian oscillator model. In Section VI, we apply our results to the multipolar chromophore-TLS coupling model. For dipolar coupling, a Padé approximant is found for the bulk spectral broadening function, and the line-shape function is calculated for various TLS densities. An alternative nonstochastic exactly solvable model for a spin bath is introduced and discussed in Appendix H.

\section{Response Functions for a Chromophore in a Boson Bath}

We consider a chromophore with two electronic states, a ground state $|g\rangle$ and an excited state $|e\rangle$, described by a Hamiltonian

$$
\hat{H}=|g\rangle \hat{H}_{g}\langle g|+| e\rangle\left(\omega_{e g}^{0}+\hat{H}_{e}\right)\langle e|
$$

Here $\hat{H}_{g}$ and $\hat{H}_{e}$ operate in the space spanned by nuclear (intermolecular and solvent) coordinates, and $\omega_{e g}^{0}$ is the unperturbed chromophore transition frequency. The electronic transition dipole operator is given by

$$
\hat{V}=|g\rangle \hat{V}_{g e}\langle e|+| e\rangle \hat{V}_{e g}\langle g|
$$

The collective bath coordinate

$$
U \equiv \hat{H}_{e}-\hat{H}_{g}
$$

represents the coupling of the chromophore to its environment and is responsible for spectral shifts and broadenings.

The linear response function $S^{(1)}(t)$ is defined through the polarization $P^{(1)}(\boldsymbol{r}, t)$, expanded to first order in the radiation field $E(\boldsymbol{r}, t)$ :

$$
P^{(1)}(\boldsymbol{r}, t)=\int_{0}^{\infty} \mathrm{d} t_{1} S^{(1)}\left(t_{1}\right) E\left(\mathbf{r}, t-t_{1}\right)
$$

Using the second-order cumulant expansion, we obtain

$$
S^{(1)}(t)=\frac{\mathrm{i}}{\hbar} \theta(t)\left[J(t)-J^{*}(t)\right]
$$

with

$$
J(t)=\exp \left(-\mathrm{i} \omega_{e g} t\right) \exp [-g(t)]
$$

and $\omega_{e g}$ is the renormalized transition frequency:

$$
\omega_{e g}=\omega_{e g}^{0}+\left\langle U \rho_{g}\right\rangle
$$

The spectral broadening function $g(t)$ is expressed in terms of the spectral density $C^{\prime \prime}(\omega)$,

$$
\begin{aligned}
& g(t)= \\
& -\frac{1}{2 \pi} \int_{-\infty}^{\infty} \mathrm{d} \omega \frac{C^{\prime \prime}(\omega)}{\omega^{2}}[1+\operatorname{coth}(\beta \hbar \omega / 2)]\left[\mathrm{e}^{-\mathrm{i} \omega t}+\mathrm{i} \omega t-1\right]
\end{aligned}
$$

where

$$
\begin{gathered}
C^{\prime \prime}(\omega)=-\mathrm{i} \int_{-\infty}^{\infty} \mathrm{d} t \mathrm{e}^{\mathrm{i} \omega t} C^{\prime \prime}(t) \\
C^{\prime \prime}(t)=-\frac{1}{2 \hbar^{2}}\left[\left\langle U(t) U(0) \rho_{g}\right\rangle-\left\langle U(0) U(t) \rho_{g}\right\rangle\right]
\end{gathered}
$$

Here $\rho_{g}$ is the equilibrium ground-state density matrix:

$$
\rho_{g}=\frac{|g\rangle\langle g| \exp \left(-\beta \hat{H}_{g}\right)}{\operatorname{Tr}\left[\exp \left(-\beta \hat{H}_{g}\right)\right]}
$$

To third order in the radiation field, the polarization reads

$$
\begin{aligned}
P^{(3)}(\mathbf{r}, t)=\int_{0}^{\infty} \mathrm{d} t_{3} \int_{0}^{\infty} \mathrm{d} t_{2} \int_{0}^{\infty} \mathrm{d} t_{1} S^{(3)}\left(t_{3}, t_{2}, t_{1}\right) \times \\
E\left(\mathbf{r}, t-t_{1}\right) E\left(\mathbf{r}, t-t_{2}-t_{1}\right) E\left(\mathbf{r}, t-t_{3}-t_{2}-t_{1}\right)
\end{aligned}
$$

Here the response function $S^{(3)}\left(t_{3}, t_{2}, t_{1}\right)$ is given by a sum of four terms:

$$
\begin{aligned}
& S^{(3)}\left(t_{3}, t_{2}, t_{1}\right)= \\
& \quad\left(\frac{\mathrm{i}}{\hbar}\right)^{3} \theta\left(t_{1}\right) \theta\left(t_{2}\right) \theta\left(t_{3}\right) \sum_{\alpha=1}^{4}\left[R_{\alpha}\left(t_{3}, t_{2}, t_{1}\right)-R_{\alpha}^{*}\left(t_{3}, t_{2}, t_{1}\right)\right]
\end{aligned}
$$

with

$$
\begin{aligned}
& R_{1}^{\mathrm{BO}}\left(t_{3}, t_{2}, t_{1}\right)=\exp \left[-g\left(t_{1}\right)-g^{*}\left(t_{2}\right)-g^{*}\left(t_{3}\right)+g\left(t_{1}+t_{2}\right)+\right. \\
& \left.g^{*}\left(t_{2}+t_{3}\right)-g\left(t_{1}+t_{2}+t_{3}\right)\right] \\
& R_{2}^{\mathrm{BO}}\left(t_{3}, t_{2}, t_{1}\right)=\exp \left[-g^{*}\left(t_{1}\right)+g\left(t_{2}\right)-g^{*}\left(t_{3}\right)-g *\left(t_{1}+t_{2}\right)-\right. \\
& \left.g\left(t_{2}+t_{3}\right)+g *\left(t_{1}+t_{2}+t_{3}\right)\right] \\
& R_{3}^{\mathrm{BO}}\left(t_{3}, t_{2}, t_{1}\right)=\exp \left[-g^{*}\left(t_{1}\right)+g *\left(t_{2}\right)-g\left(t_{3}\right)-g *\left(t_{1}+t_{2}\right)-\right. \\
& \left.g *\left(t_{2}+t_{3}\right)+g *\left(t_{1}+t_{2}+t_{3}\right)\right] \\
& R_{4}^{\mathrm{BO}}\left(t_{3}, t_{2}, t_{1}\right)=\exp \left[-g\left(t_{1}\right)-g\left(t_{2}\right)-g\left(t_{3}\right)+g\left(t_{1}+t_{2}\right)+\right. \\
& \left.g\left(t_{2}+t_{3}\right)-g\left(t_{1}+t_{2}+t_{3}\right)\right]
\end{aligned}
$$

These results are exact for the spin-boson Hamiltonian which represents the Brownian oscillator model (Appendix A). For this model, $C^{\prime \prime}(\omega)$ (eq A6) is independent of temperature, and the entire temperature dependence of $g(t)$ comes from the coth factor in eq 2.8. This will be compared in Section V with the TLS model.

The linear response function can always be recast in the form of eqs 2.5-2.7 and expressed in terms of a single spectral density. Equation 2.14 shows that for the boson bath the same spectral density determines the third-order response. This is not generally the case for other models, ${ }^{3}$ where more information is needed in order to calculate $S^{(3)}$. A simple example is the inhomogeneous cumulant expansion ${ }^{3}$ where $g(t)$ depends on 
some system parameters, and the response functions $S^{(1)}(2.5)$ and $S^{(3)}$ (2.14) are averaged over the distribution of these parameters.

\section{Response Functions for a Chromophore in a Stochastic Spin Bath}

We assume that our two-state chromophore is coupled to a collection of two-level systems (TLSs) (labeled by the index $j$ ). The transition frequency of the chromophore depends on the state of the TLS, described by a time-dependent occupation variable $\xi_{j}: \xi_{j}=0$ if the TLS is in its ground state, and $\xi_{j}=1$ if the TLS is excited. Assuming that the chromophore's transition frequency is changed by the amount $v_{j}$ when $\xi_{j}$ is changed from 0 to 1 , then the collective bath coordinate (eq $2.3)$ is

$$
U=\sum_{j} v_{j} \xi_{j}
$$

The populations of the ground and excited TLS states $P_{j}^{0}(t)$ and $P_{j}^{1}(t)$ satisfy the rate equations

$$
\left(\begin{array}{l}
\dot{P}_{j}^{0}(t) \\
\dot{P}_{j}^{1}(t)
\end{array}\right)=\left(\begin{array}{ll}
-k_{j}^{u} & k j^{d} \\
k_{j}^{u} & -k_{j}^{d}
\end{array}\right)\left(\begin{array}{l}
P_{j}^{0}(t) \\
P_{j}^{1}(t)
\end{array}\right)
$$

where $k_{j}^{u}$ and $k_{j}^{d}$ are the rate constants for up and down transitions for the $j$ th TLS. In thermal equilibrium, one obtains the probability $P_{j}^{k}$ of finding the $j$ th TLS in state $k(k=0,1)$ :

$$
P_{j}^{k}=p_{j} \delta_{k, 1}+\left(1-p_{j}\right) \delta_{k, 0}
$$

Here

$$
p_{j}=\left[\exp \left(\epsilon_{j} / k_{\mathrm{B}} T\right)+1\right]^{-1}
$$

and $\epsilon_{j}$ is the energy difference of the $j$ th TLS. We introduce the dimensionless parameter

$$
\kappa_{\mathrm{j}} \equiv \epsilon_{j} / k_{\mathrm{B}} T
$$

For this model, the time-domain response function $J(t)$ is given by the product of the individual response functions $J_{j}(t)$ (see Appendix B):

$$
J(t)=\exp \left(-\mathrm{i} \omega_{e g} t\right) \Pi_{j} J_{j}(t)
$$

where

$$
J_{j}(t)=\theta(t) \mathrm{e}^{-\left(b_{j} / 2\right) t}\left[\cosh \left(\Omega_{j} t\right)+\frac{2 d_{j}-b_{j}}{2 \Omega_{j}} \sinh \left(\Omega_{j} t\right)\right]
$$

with

$$
\begin{gathered}
\Omega_{j}=\sqrt{\frac{b_{j}^{2}}{4}-c_{j}}=\sqrt{\frac{K_{j}^{2}}{4}-\frac{v_{j}^{2}}{4}-\mathrm{i}\left(p_{j}-\frac{1}{2}\right) v_{j} K_{j}} \\
b_{j}=\mathrm{i} v_{j}+K_{j} \\
c_{j}=\mathrm{i} v_{j} K_{j} p_{j} \\
d_{j}=K_{j}+\mathrm{i} v_{j}\left(1-p_{j}\right) \\
K_{j}=k_{j}^{u}+k_{j}^{d}
\end{gathered}
$$

The third-order response function can be expressed in terms of the Liouville-space Green functions given in Appendix D. Third-order response functions corresponding to $R_{2}$ and $R_{3}$ were first calculated for spin echoes by Klauder and Anderson (twoand three-pulse, short times) and Mims (two-pulse, long times $^{36,57}$ for electronic spin resonance. High-temperature results for arbitrary times were obtained by $\mathrm{Hu}$ and Hartmann ${ }^{37}$ for both two- and three-pulse echoes. Generalizations to finite temperatures were later given by $\mathrm{Hu}$ and Walker. ${ }^{38}$ Utilizing Pauli matrices, Huber extended the two-pulse echo results of $\mathrm{Hu}$ and Hartmann (dipolar) to multipolar interactions $\left(1 / r^{n}\right) .{ }^{39}$ Suárez and Silbey recently derived the most general form of two- and three-pulse photon-echo responses valid for multipolar interactions and arbitrary temperatures. ${ }^{40}$ However, the full response function including $R_{1}$ and $R_{4}$ in addition to $R_{2}$ and $R_{3}$ has not been calculated. In this section, we derive the complete third-order response function. In the special case of the photonecho response our results reproduce earlier calculations. ${ }^{37,40}$

Since the TLS dynamics are not affected by whether the chromophore is in the ground state or excited state, this model misses the Stokes shift. ${ }^{3}$ Therefore $R_{1 j}=R_{4 j}$, and $R_{2 j}=R_{3 j}$. The time-domain third-order response function $S^{(3)}\left(t_{3}, t_{2}, t_{1}\right)$ is given by eq (2.12) (cf. Appendix D):

$$
\begin{aligned}
& R_{1}\left(t_{3}, t_{2}, t_{1}\right)=\Pi_{j} R_{1 j}\left(t_{3}, t_{2}, t_{1}\right) \\
& R_{2}\left(t_{3}, t_{2}, t_{1}\right)=\Pi_{j} R_{2 j}\left(t_{3}, t_{2}, t_{1}\right)
\end{aligned}
$$

with

$$
\begin{gathered}
R_{1 j}\left(t_{3}, t_{2}, t_{1}\right)=\mathrm{e}^{-\mathrm{i}\left(\omega_{e g}+v_{j} / 2\right)\left(t_{1}+t_{3}\right)-\left(K_{j} / 2\right)\left(t_{1}+t_{3}\right)} \\
{\left[\left(1-p_{j}\right) F_{j}\left(t_{3}\right) F_{j}\left(t_{1}\right)+p G_{j}\left(t_{1}\right) G_{j}\left(t_{1}\right)+\right.} \\
\left.\left(1-\mathrm{e}^{-K_{j} t_{2}}\right) \frac{p_{j}\left(1-p_{j}\right) v_{j}^{2}}{\Omega_{j}^{2}} \sinh \left(\Omega_{j} t_{3}\right) \sinh \left(\Omega_{j} t_{1}\right)\right] \\
R_{2 j}\left(t_{3}, t_{2}, t_{1}\right)=\mathrm{e}^{-\mathrm{i}\left(\omega_{e g}+v_{j} / 2\right)\left(t_{1}-t_{3}\right)-\left(K_{j} / 2\right)\left(t_{1}+t_{3}\right)} \\
{\left[\begin{array}{l}
\left(1-p_{j}\right) F_{j}\left(t_{3}\right) F_{j}^{*}\left(t_{1}\right)+p G_{j}\left(t_{1}\right) G_{j}^{*}\left(t_{1}\right)- \\
\left.\left(1-\mathrm{e}^{-K_{j} t_{2}}\right) \frac{p_{j}\left(1-p_{j}\right) v_{j}^{2}}{\left|\Omega_{j}\right|^{2}} \sinh \left(\Omega_{j} t_{3}\right) \sinh \left(\Omega_{j}^{*} t_{1}\right)\right]
\end{array}\right]}
\end{gathered}
$$

where

$$
\begin{aligned}
F_{j}(t) & =\cosh \left(\Omega_{j} t\right)+\frac{b_{j}}{2 \Omega_{j}} \sinh \left(\Omega_{j} t\right) \\
G_{j}(t) & =\cosh \left(\Omega_{j} t\right)+\frac{b_{j}^{*}}{2 \Omega_{j}} \sinh \left(\Omega_{j} t\right)
\end{aligned}
$$

Only $R_{2}$ is relevant to photo-echo experiments. The corresponding frequency-domain response functions are given in Appendix C.

In the high-temperature limit $\left(\kappa_{j} \ll 1\right.$ and $\left.p_{j}=1 / 2\right)$, these response functions can be simplified: 

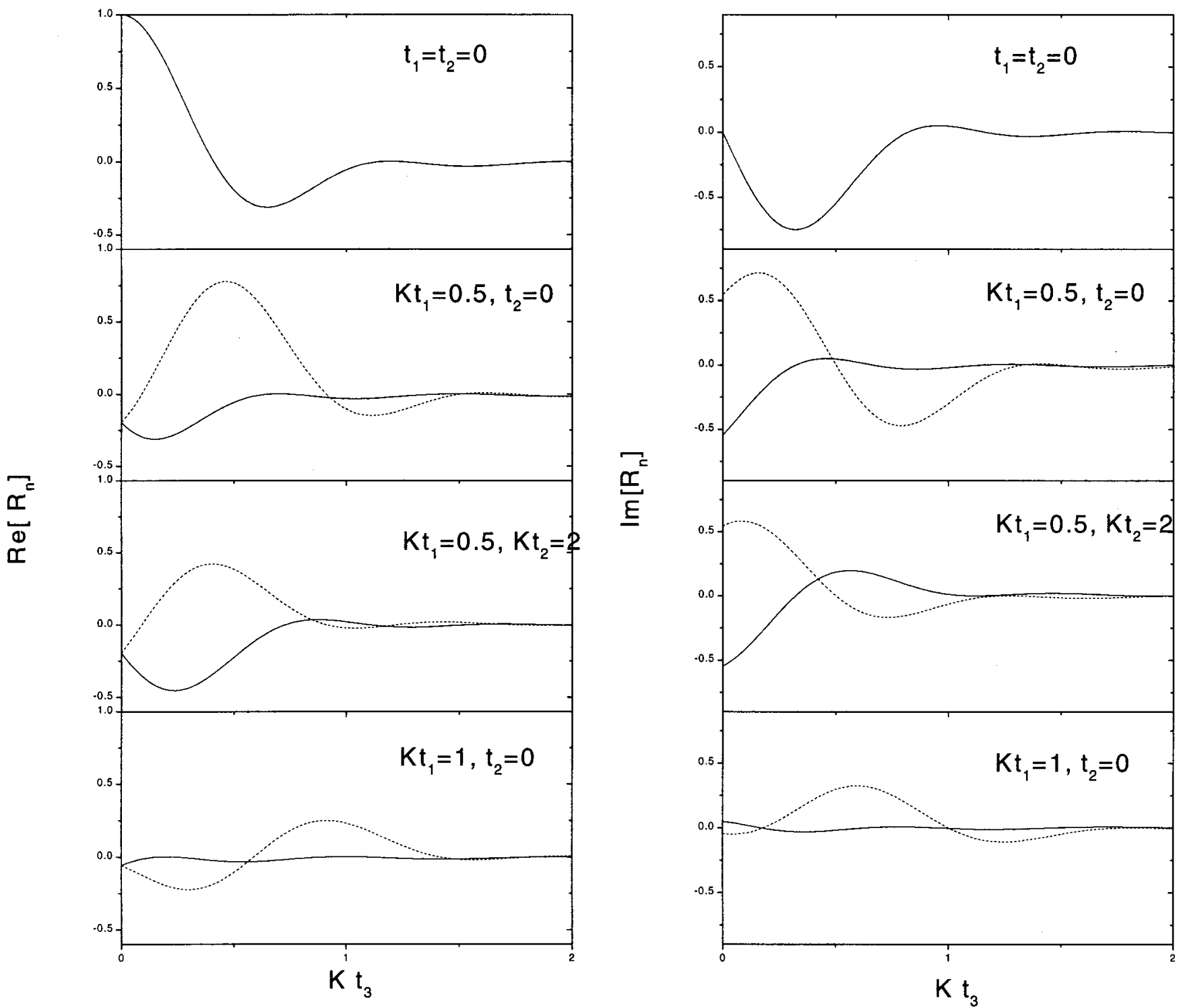

Figure 1. Comparison of $R_{1}\left(t_{3}, t_{2}, t_{1}\right)$ and $R_{2}\left(t_{3}, t_{2}, t_{1}\right)$ for a chromophore interacting with a bath of four TLSs with identical jump rates but randomly chosen coupling strengths $v(v / k=0.60,1.38,2.31,3.37) . p=0.5$. Left column, real parts. Right column, imaginary parts. At $t_{1}=0$, the two response functions have no $t_{2}$ dependence and are identical (top panel). The decay of the response functions with $t_{2}$ is illustrated in the two middle panels.]

$$
\begin{aligned}
& R_{1 j}\left(t_{3}, t_{2}, t_{1}\right)= \frac{1}{2} \mathrm{e}^{-\mathrm{i}\left(\omega_{e g}+v_{j} / 2\right)\left(t_{1}+t_{3}\right)-\left(K_{j} / 2\right)\left(t_{1}+t_{2}+t_{3}\right)} \times \\
& {\left[\sinh \left(\frac{K_{j} t_{2}}{2}\right)\left(F_{j}\left(t_{3}\right) G_{j}\left(t_{1}\right)+G_{j}\left(t_{3}\right) F_{j}\left(t_{1}\right)\right)+\right.} \\
&\left.\cosh \left(\frac{K_{j} t_{2}}{2}\right)\left(F_{j}\left(t_{3}\right) F_{j}\left(t_{1}\right)+G_{j}\left(t_{3}\right) G_{j}\left(t_{1}\right)\right)\right] \\
& R_{2 j}\left(t_{3}, t_{2}, t_{1}\right)= \frac{1}{2} \mathrm{e}^{\mathrm{i}\left(\omega_{e g}+v_{j} / 2\right)\left(t_{1}-t_{3}\right)-\left(K_{j} / 2\right)\left(t_{1}+t_{2}+t_{3}\right)} \times \\
& {\left[\sinh \left(\frac{K_{j} t_{2}}{2}\right)\left(F_{j}\left(t_{3}\right) \mathrm{G}_{j}^{*}\left(t_{1}\right)+G_{j}\left(t_{3}\right) F_{j}^{*}\left(t_{1}\right)\right)+\right.} \\
&\left.\cosh \left(\frac{K_{j} t_{2}}{2}\right)\left(F_{j}\left(t_{3}\right) F_{j}^{*}\left(t_{1}\right)+G_{j}\left(t_{3}\right) G_{j}^{*}\left(t_{1}\right)\right)\right]
\end{aligned}
$$

$S^{(3)}\left(t_{3}, t_{2}, t_{1}\right)$ can be applied to various third-order experiments in single-molecule and bulk spectroscopy. Most of the earlier work had focused on the three-pulse echo technique in the limit of large inhomogeneous broadening. In that case we only need the response function $R_{2}$ for $t_{1}=t_{3}$, and we have

$$
\begin{gathered}
R_{2 j}\left(t_{1}, t_{2}, t_{1}\right)=\frac{\exp \left[-\mathrm{i}\left(2 \omega_{e g}+v_{j}\right) t_{1}-k_{j} t_{1}\right]}{4 \bar{\Omega}_{j}^{2}}\left[K_{j}^{2} \cos \left(2 \bar{\Omega}_{j} t_{1}\right)-\right. \\
\left.v_{j}^{2}+2 K_{j} \bar{\Omega}_{j} \sin \left(2 \bar{\Omega}_{j} t_{1}\right)+\left(1-\mathrm{e}^{-K_{j} t_{2}}\right) \sin ^{2}\left(\bar{\Omega}_{j} t_{1}\right)\right]
\end{gathered}
$$

with

$$
\bar{\Omega}_{j}=\frac{1}{2} \sqrt{v_{j}^{2}-K_{J}^{2}}
$$

which agrees with results of $\mathrm{Hu}$ and Hartmann ${ }^{37}$ and Suárez and Silbey. ${ }^{40}$

In Figure 1 , we compare $R_{1}\left(t_{1}, t_{2}, t_{3}\right)$ and $R_{2}\left(t_{1}, t_{2}, t_{3}\right)$ for a chromophore interacting with a bath of four TLSs with identical rates and different coupling strengths $v(v / K=0.60,1.38,2.31$, 3.37). At $t_{1}=0$, the two response functions have no $t_{2}$ dependence and are therefore identical. However, for $K t_{1}=$ $0.5, t_{2}=0$ and $K t_{1}=1, t_{2}=0$, the two are very different. The decay of the response functions as a function of $t_{2}$ is revealed by comparing the responses for $t_{2}=0$ and $K t_{2}=2$ at $K t_{1}=0.5$ (the two middle panels). 


\section{Cumulant Expansion for Many TLSs}

To calculate $J(t)$ for a large ensemble of TLSs, we first recast it in the form of eq (2.5) with

$$
\begin{aligned}
& g(t)= \\
& -\sum_{j} \ln \left\{1+\left[\mathrm{e}^{-\left(b_{j} / 2\right) t}\left(\cosh \left(\Omega_{j} t\right)+\frac{2 d_{j}-b_{j}}{2 \Omega_{j}} \sinh \left(\Omega_{j} t\right)\right)-1\right]\right\}
\end{aligned}
$$

Assuming that each of the TLSs makes a very small contribution to the line shape, we have

$$
\begin{array}{r}
g(t)= \\
\sum_{j}\left[1-\mathrm{e}^{-\left(b_{j} / 2\right) t}\left(\cosh \left(\Omega_{j} t\right)+\frac{2 d_{j}-b_{j}}{2 \Omega_{j}} \sinh \left(\Omega_{j} t\right)\right)\right]= \\
\sum_{j}\left[1-J_{j}(t)\right]
\end{array}
$$

Similarly, eq (3.6) takes the form

$$
J(t)=\exp \left(-\mathrm{i} \omega_{e g} t\right) \mathrm{e}^{-N\left[1-\left\langle J_{j}(t)\right\rangle\right]}
$$

where

$$
\left\langle J_{j}(t)\right\rangle=\frac{1}{N} \sum_{j} J_{j}(t)
$$

Measured optical properties of chromophores need to be averaged over many chromophores embedded in the host matrixes. Each chromophore has a distinct coupling to the TLS. Therefore an average over the TLS configuration with respect to the chromophore needs to be performed. This averaging is eliminated for the single-molecule spectroscopy (SMS). Therefore the SMS response function is given by eq (3.6) and eq (3.7).

Introducing the distribution function $n(k, v, E)$ for the TLS parameters $K_{j}, v_{j}$, and $p_{j}$, we write the configurational average as

$$
\langle J\rangle_{c} \equiv \int \mathrm{d} v \mathrm{~d} k \mathrm{~d} E J n(k, v, E)
$$

with the normalization

$$
\int \mathrm{d} v \mathrm{~d} k \mathrm{~d} E n(k, v, E)=1
$$

This is similar to our notation of averages over the TLS index j:

$$
\langle\rangle \equiv \frac{1}{N} \sum_{j}
$$

Performing the configurational average, we arrive at the bulk response function $J_{c}(t)$ :

$$
J_{c}(t)=\exp \left(-\mathrm{i} \omega_{e g} t\right) \Pi_{j}\left\langle J_{j}(t)\right\rangle_{c}
$$

where the angular bracket denotes the configurational average. The averaging allows the product $\Pi_{j}$ to be replaced by the $N$ th power ( $N$ is the total number of TLSs):

$$
J_{c}(t)=\exp \left(-\mathrm{i} \omega_{e g} t\right)\left(\left\langle J_{j}(t)\right\rangle_{c}\right)^{N}
$$

while the $N \rightarrow \infty$ limit gives

$$
J_{c}(t)=\exp \left(-\mathrm{i} \omega_{e g} t\right) \exp \left[-N\left(1-\left\langle J_{j}(t)\right\rangle_{c}\right)\right]
$$

The configurational average \langle\rangle$_{c}$ contains summations over positions of the TLS with respect to the chromophore. It is obvious that the single-chromophore response function $J(t)$ becomes the bulk response function $J_{c}(t)$ once $\left\langle J_{j}(t)\right\rangle$ is replaced by the configurationally averaged $\left\langle J_{j}(t)\right\rangle_{c}$. $\left\langle J_{j}(t)\right\rangle$ is a sum over $j$ which reflects the random structure of a particular realization of the chromophore-TLS system, while $\left\langle J_{j}(t)\right\rangle_{c}$ is given by an integral over the configurational coordinates. Therefore only the bulk properties of the TLS and their coupling to the chromophore are reflected in $\left\langle J_{j}(t)\right\rangle_{c}$.

In evaluating the bulk response functions, two averages need to be performed if one views the evaluation of $J_{j}(t)$ as an average over stochastic trajectories. In general, one can reverse the order of the configurational average and the average over the stochastic trajectories denoted by the angular bracket \langle\rangle$_{s}$. If the configurational average is carried out first, we obtain

$$
J_{c}(t)=\exp \left(-\mathrm{i} \omega_{e g} t\right)\left[1-\frac{1}{N} N\left\langle\left\langle 1-\mathrm{e}^{\mathrm{i} v_{j}} \int_{0}^{t} \xi_{j} \mathrm{~d} \tau\right\rangle_{c}\right\rangle_{s}\right]^{N}
$$

In the limit of large $N$, we have

$$
J_{c}(t)=\exp \left(-\mathrm{i} \omega_{e g} t\right) \exp \left[-N\left\langle\left\langle 1-\mathrm{e}^{\mathrm{i} v_{j} \int_{0}^{t} \xi_{j} \mathrm{~d} \tau}\right\rangle_{c}\right\rangle_{s}\right]
$$

The approach of the stochastic average, which is equivalent to our derivation of response functions via Green's functions, can be simulated by the Monte-Carlo techniques. ${ }^{58}$

The configurationally averaged line-shape function $g_{c}(t)$ assumes the form

$$
\frac{1}{N} g_{c}(t)=\left\langle\ln \left[\mathrm{e}^{-(b / 2) t}\left(\cosh (\Omega t)+\frac{2 d-b}{2 \Omega} \sinh (\Omega t)\right)\right]\right\rangle_{c}
$$

where $b, d$, and $\Omega$ are functions of $k, v$, and $p$ as given by eqs $3.8-3.12$. We shall discuss the properties of $g_{c}(t)$ in several different limits.

Assuming that $n(k, v, E)$ is nonzero only in the regime $v \ll k$, we can expand eq 4.13 in powers of $v / k$. The correlation function $C(t)$ to fifth order reads

$$
\begin{aligned}
& C(t)=\left\langle v^{2} p(1-p) \mathrm{e}^{-k t}\right\rangle_{c}+i\left\langle v^{3} p(1-p)(1-2 p) t \mathrm{e}^{-k t}\right\rangle_{c}+ \\
&\left\langlev ^ { 4 } \frac { p ( 1 - p ) } { 2 k ^ { 2 } } \mathrm { e } ^ { - k t } \left[ 4 p(1-p)\left(\mathrm{e}^{-k t}+k t-1\right)-\right.\right. \\
&\left.\left.(1-2 p)^{2} k^{2} t^{2}\right]\right\rangle_{c}+i\left\langle v^{5} \frac{p(1-p)(1-2 p)}{k^{3}} \mathrm{e}^{-k t} \times\right. \\
&\left.\left\{\frac{1}{6}(1-2 p)^{2} k^{3} t^{3}-2 p(1-p)\left(k^{2} t^{2}-2\left(\mathrm{e}^{-k t}+k t-1\right)\right]\right\}\right\rangle_{c}
\end{aligned}
$$

The line-broadening function to fourth order is 


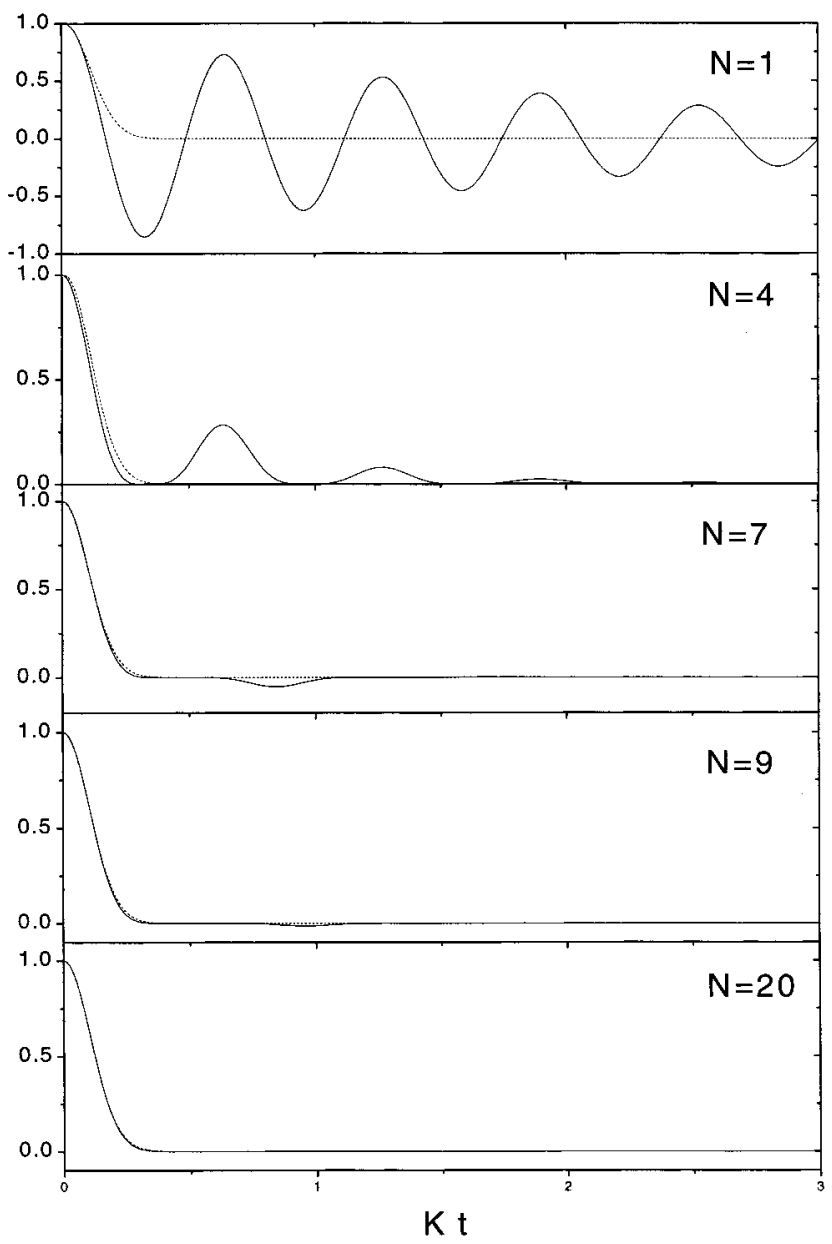

Figure 2. Time-domain linear response functions $J(t)$ (eq (3.6)) for $\alpha$ $=10$ and from top down, $N=1,4,7,9,20$. The dashed curve is the second-order cumulant.]

$$
\begin{gathered}
\left\langle U \rho_{g}\right\rangle=\langle p v\rangle_{c} \\
\frac{1}{N} g_{c}(t)=\left\langle\frac{p(1-p) v^{2}}{k^{2}}\left(\mathrm{e}^{-k t}+k t-1\right)\right\rangle_{c}- \\
\mathrm{i}\left\langle\frac{p(p-1)(2 p-1) v^{3}}{k^{3}}\left[(k t+2) \mathrm{e}^{-k t}+(k t-2)\right]\right\rangle_{c}+ \\
\left\langle\frac{p(1-p) v^{4}}{2 k^{4}}[p(1-p)(10 k t-29)-2(k t-3)-\right. \\
\left(k^{2} t^{2}+4 k t+6-4 p(1-p)\left(k^{2} t^{2}+5 k t+7\right)\right) \mathrm{e}^{-k t}+ \\
\left.\left.p(1-p) \mathrm{e}^{-2 k t}\right]\right\rangle_{c}
\end{gathered}
$$

Note that $g_{c}(t)$ is an analytic function of $k$; as $k \rightarrow 0$, the timedependent factor in the $n$th term behaves as $k^{n}$, which cancels the $k^{-n}$ factors, and $g_{c}(t)$ remains finite. In the high-temperature regime $\kappa \ll 1$, the third-order term vanishes, and we obtain

$$
\begin{gathered}
\left\langle U \rho_{g}\right\rangle=\left\langle\frac{v}{2}\right\rangle_{c} \\
\frac{1}{N} g_{c}(t)=\left\langle\frac{v^{2}}{4 k^{2}}\left(\mathrm{e}^{-k t}+k t-1\right)\right\rangle_{c}+ \\
\left\langle\frac{v^{4}}{8 k^{4}}\left[\frac{k t}{2}-\frac{5}{4}-(k t+1) \mathrm{e}^{-k t}-\frac{1}{4} \mathrm{e}^{-2 k t}\right]\right\rangle_{c}
\end{gathered}
$$

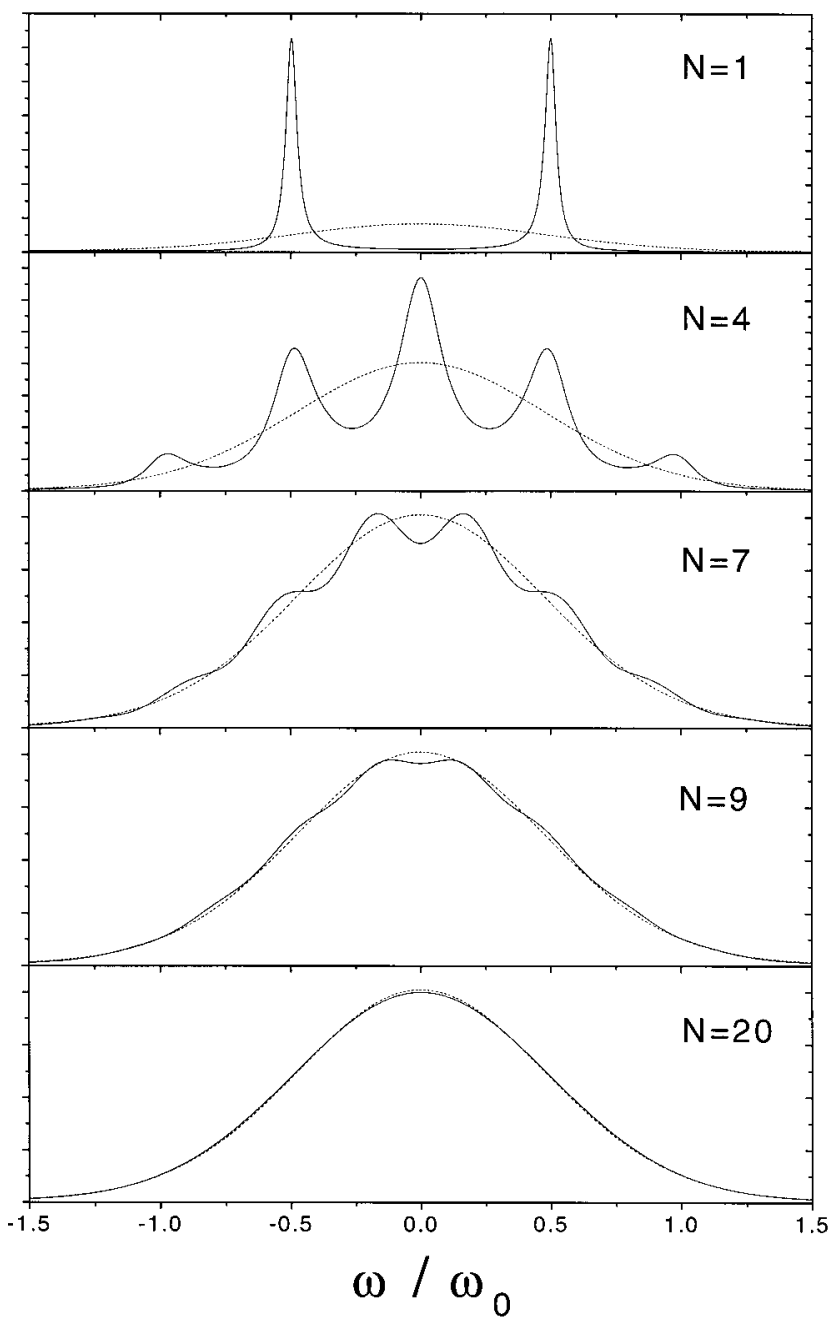

Figure 3. Absorption line-shapes $\sigma(\omega)$ (eq (4.21)) for $\alpha=10$ and from top down, $N=1,4,7,9,20$ (solid). $\omega_{0} 2=N v^{2}$. The dashed curve is the second-order cumulant.]

To second order in $v / k$, we may rewrite eq 4.16 as

$$
\frac{1}{N} g_{c}(t)=\int \mathrm{d} k \frac{C(k)}{k^{2}}\left(\mathrm{e}^{-k t}+k t-1\right)
$$

with

$$
C(k)=\int \mathrm{d} v \mathrm{~d} E n(k, v, E) v^{2} p(1-p)
$$

Equation 4.19 recovers the stochastic oscillator model with the distribution of the rates $k$ given by the spectral density $C(k)$ of eq $4.20 .^{3}$

The linear absorption line-shape function $\sigma(\omega)$ is given by the Fourier transform of the response function $J(t)$ :

$$
\sigma(\omega)=\frac{1}{\pi} \operatorname{Re} \int_{0}^{\infty} \mathrm{d} t \mathrm{e}^{\mathrm{i} \omega t} J(t)
$$

In Figure 2, we display $J(t)$ for a fixed value of the dimensionless parameter $\alpha \equiv \sqrt{N} v / 2 k(\alpha=10)$ and increasing $N(N=1,4,7,9,20$ from top down). These are compared with the second-order cumulant (dashed). The latter only captures the short-time behavior while leaving out the longtime oscillations in the exact response functions. The corresponding line-shape functions $\sigma(\omega)$ are displayed in Figure 3. 


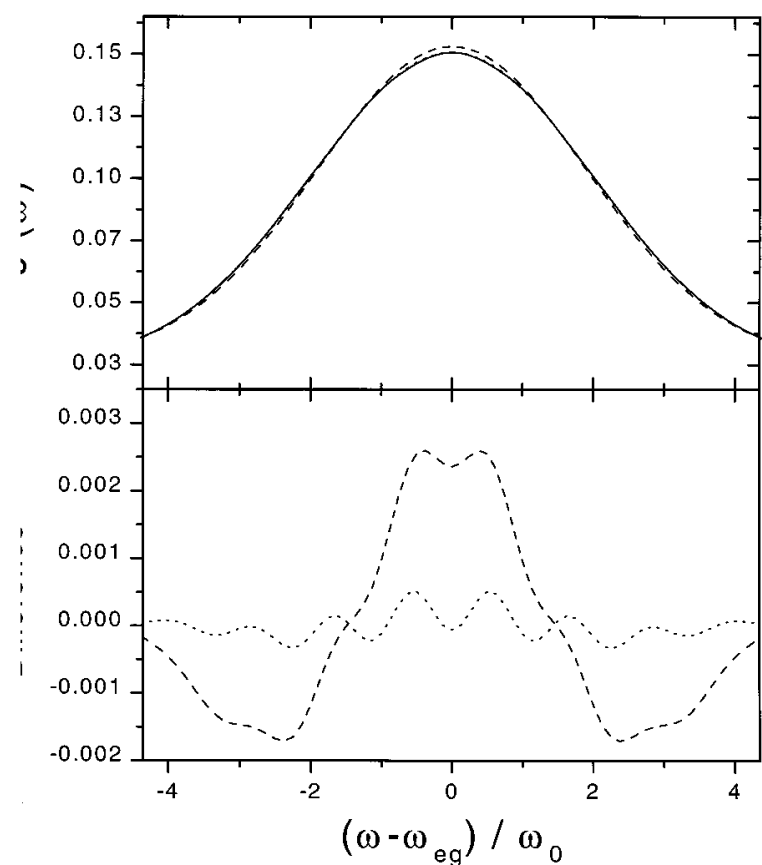

Figure 4. Comparison of various approximations of the line-shape function for $p=0.5, \alpha=10, N=12$. Upper panel: exact line-shape function $\sigma(\omega)$ (solid), second-order cumulant $\sigma^{2 \mathrm{nd}}(\omega)$ (dashed), and fourth-order cumulant $\sigma^{\text {th }}(\omega)$ (dotted, coincides with the solid curve). Lower panel: second-order $\sigma^{\text {4th }}(\omega)-\sigma(\omega)$ (solid) and fourth-order corrections $\sigma^{4 \text { th }}(\omega)-\sigma(\omega)$ (dashed).]

Here $N+1$ peaks are resolved in the line-shape functions with $N$ TLS. The second-order cumulant is recovered as the large- $N$ limit. This is expected from the central-limit theorem. In Figure 4, we compare the exact line-shape function $\sigma(\omega)$ (solid) with the second- (dashed) and fourth-order (dotted) cumulant expressions. For the parameters used $(\alpha=10, N=12)$, the secondorder cumulant works quite well, and the fourth-order correction improves its accuracy. In Figure 5 we compare the exact thirdorder response function $R_{2}\left(t_{3}, t_{2}, t_{1}\right)$ with the second- and fourthorder cumulant expressions for the same set of parameters of Figure 4, $N=12$. All curves are plotted for $K t_{1}=0.3$ and five values of $K t_{2}$ (in the top panel, from top down, $K t_{2}=0,0.1$, $0.2,0.3,0.4,0.5)$. The middle panel shows the small $(\sim 1 \%)$ difference between the second-order cumulant and the exact response function $R_{2}\left(t_{3}, t_{2}, t_{1}\right)$. The difference between the fourthorder cumulant and $R_{2}\left(t_{3}, t_{2}, t_{1}\right)$ is less than one-tenth of a percent. Let us denote by $R_{n}^{0}(n=1,2,3,4)$ the third-order response function obtained by substituting the fourth-order line-broadening function (eq 4.16) into the Brownian oscillator expression. The response function calculated using the fourth-order cumulant expansion may be recast in the form

$R_{n}\left(t_{3}, t_{2}, t_{1}\right)=R_{n}^{0}\left(t_{3}, t_{2}, t_{1}\right) \exp \left[-F_{n}\left(t_{3}, t_{2}, t_{1}\right)\right]$,

The functions $F_{n}$ represent the deviation of TLS nonlinear response functions from the form of eq 2.14. We find that to second-order in $v / k F_{n}$ vanish for all temperatures. In Appendix $\mathrm{E}$, we give $F_{n}\left(t_{3}, t_{2}, t_{1}\right)$ to fourth order in $v / k$. In the hightemperature limit $(\kappa \gg 1)$, these expressions assume a simpler form:

$$
\begin{gathered}
\begin{array}{c}
F_{1}\left(t_{3}, t_{2}, t_{1}\right)=F_{4}\left(t_{3}, t_{2}, t_{1}\right)= \\
\left\langle( \frac { v } { 2 k } ) ^ { 4 } \mathrm { e } ^ { - k t _ { 2 } } ( 1 - \mathrm { e } ^ { - k t _ { 1 } } ) ( 1 - \mathrm { e } ^ { - k t _ { 3 } } ) \left[ 2 k t_{2}+\right.\right. \\
\left.\left.\left(1-\mathrm{e}^{-k t_{2}}\right)\left(\mathrm{e}^{-k t_{1}}+\mathrm{e}^{-k t_{3}}\right)\right]\right\rangle_{c} \\
F_{2}\left(t_{3}, t_{2}, t_{1}\right)=F_{3}\left(t_{3}, t_{2}, t_{1}\right)= \\
\left\langle( \frac { v } { 2 k } ) ^ { 4 } \mathrm { e } ^ { - k t _ { 2 } } ( 1 - \mathrm { e } ^ { - k t _ { 1 } } ) ( 1 - \mathrm { e } ^ { - k t _ { 3 } } ) \left[ 2 k t_{2}+\right.\right. \\
\left.\left.\mathrm{e}^{-k t_{2}}\left(1+\mathrm{e}^{-k t_{1}-k t_{3}}\right)-\left(\mathrm{e}^{-k t_{1}}+\mathrm{e}^{-k t_{3}}\right)\right]\right\rangle_{c}
\end{array}
\end{gathered}
$$

\section{Temperature-Dependent Response of the Spin and the Boson Bath}

We note that the second-order contribution to $C(t)$ (eq 4.14) is real. Using the fluctuation-dissipation theorem, we can compute its imaginary part as well. ${ }^{3}$ At finite temperatures, the line-shape function for the spin bath $g^{\mathrm{TLS}}(t)$ can be calculated from

$$
\begin{aligned}
g^{\mathrm{TLS}}(t)=\frac{1}{2 \pi} \int_{-\infty}^{\infty} \mathrm{d} \omega \frac{1-\cos (\omega t)}{\omega^{2}} C^{\prime}(\omega)- \\
\frac{i}{2 \pi} \int_{-\infty}^{\infty} \mathrm{d} \omega \frac{\sin (\omega t)-\omega t}{\omega^{2}} \tanh \frac{\beta \hbar \omega}{2} C^{\prime}(\omega)
\end{aligned}
$$

where

$$
C^{\prime}(\omega)=2 p(1-p) \frac{v^{2} \Lambda}{\omega^{2}+\Lambda^{2}}
$$

Here $\Lambda=K$ stands for the flipping rate of the TLS. The temperature dependence of $g^{\mathrm{TLS}}$ enters through $p, \Lambda$, and the tanh factor. We shall compare this result with the boson bath line-shape function $g^{\mathrm{BO}}(t)$

$$
\begin{array}{r}
g^{\mathrm{BO}}(t)=\frac{1}{2 \pi} \int_{-\infty}^{\infty} \mathrm{d} \omega \frac{1-\cos (\omega t)}{\omega^{2}} \operatorname{coth}(\beta \hbar \omega / 2) C^{\prime \prime}(\omega)+ \\
\frac{i}{2 \pi} \int_{-\infty}^{\infty} \mathrm{d} \omega \frac{\sin (\omega t)-\omega t}{\omega^{2}} C^{\prime \prime}(\omega)
\end{array}
$$

For the overdamped Brownian oscillator model, the spectral density function $C^{\prime \prime}(\omega)$ takes the form

$$
C^{\prime \prime}(\omega)=2 \lambda \frac{\omega \Lambda}{\omega^{2}+\Lambda^{2}}
$$

For this model, $\lambda$ and $\Lambda$ are both temperature independent, and the only temperature dependence enters through the coth factor. Expressions suitable for numerical calculations of eqs 5.1 and 5.3 are presented in Appendix F.

In Figure 6, we compare the temperature dependences of $C^{\prime}(\omega)$ and $C^{\prime \prime}(\omega)$ for the Brownian oscillator and the TLS models assuming that $\Lambda$ is temperature independent. For the latter, $C^{\prime}(\omega)$ increases with temperature and levels off for high temperatures, and $C^{\prime \prime}(\omega)$ peaks at $k_{\mathrm{B}} T=\epsilon$. Both $C^{\prime}(\omega)$ and $C^{\prime \prime}(\omega)$ vanish at zero temperature. For the Brownian oscillator model, $C^{\prime \prime}(\omega)$ is temperature independent, and $C^{\prime}(\omega)$ increases monotonically with temperature at high temperatures. At low temperatures, $C^{\prime}(\omega)$ has a dip at $\omega=0$. Therefore, in the Brownian oscillator model, $C^{\prime}(\omega)\left(C^{\prime \prime}(\omega)\right)$ dominates at high (low) temperatures. In the weak coupling regime, we can utilize eq 2.14 to obtain $R_{n}\left(t_{3}, t_{2}, t_{1}\right)$ with $n=1,2,3,4$. Since now the 


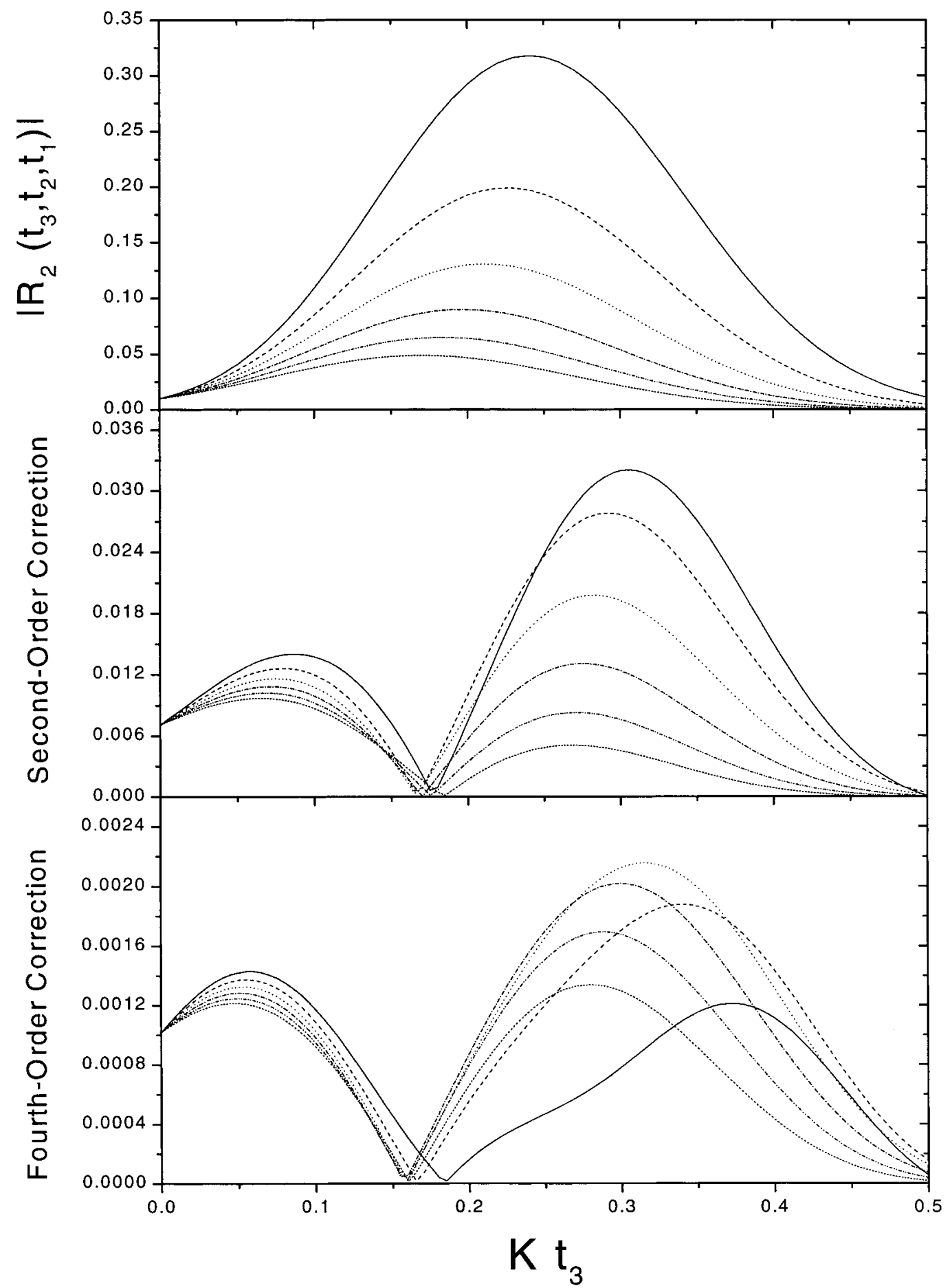

Figure 5. Comparison of the exact third response function $R_{2}\left(t_{3}, t_{2}, t_{1}\right)$ and the its second- and fourth-order cumulants $\left(R_{2}^{2 \text { nd }}\left(t_{3}, t_{2}, t_{1}\right)\right.$ and $R_{2}^{4 \text { th }}\left(t_{3}, t_{2}, t_{1}\right)$, respectively) for $p=0.5, \alpha=10, N=12$. Upper panel: $\left|R_{2}\left(t_{3}, t_{2}, t_{1}\right)\right|$. Middle panel: $\left|R_{2}\left(t_{3}, t_{2}, t_{1}\right)-R_{2}^{2 \text { nd }}\left(t_{3}, t_{2}, t_{1}\right)\right|$. Lower panel: $\mid R_{2}\left(t_{3}, t_{2}, t_{1}\right)-$ $R_{2}^{4 \text { th }}\left(t_{3}, t_{2}, t_{1}\right) \mid . K t_{1}=0.3$. Five values of $K t_{2}$ are selected: 0.0 (solid), 0.1 (dashed), 0.2 (dotted), 0.3 (dash-dotted), 0.4 (dash-double-dotted), 0.5 (short-dashed).]

imaginary part of $g(t)$ is included and the Stokes shift has been accounted for, $R_{1}\left(t_{3}, t_{2}, t_{1}\right)\left(R_{2}\left(t_{3}, t_{2}, t_{1}\right)\right)$ is no longer identical to $R_{4}\left(t_{3}, t_{2}, t_{1}\right)\left(R_{3}\left(t_{3}, t_{2}, t_{1}\right)\right)$.

In Figure 7, we show the absorption line shapes of the Brownian oscillator model. For a fixed temperature, e.g., $k_{\mathrm{B}} T=\lambda / 4$, the absorption width increases with decreasing $\Lambda$ (slower bath) in the BO model. At the same time, the line shape approaches a Gaussian in the static limit, and the Stokes shifts tends to $2 \lambda$. For the TLS model, however, the absorption width decreases with decreasing $\Lambda$, and the slow modulation limit of the absorption line shapes is a Lorentzian. In Figure 8, absorption line shapes of the extended TLS model are shown for six values of relaxation rate $\Lambda$ and $T / \epsilon=6$ (left panel). At low temperatures, the TLS line shape is neither a Lorentzian nor a Gaussian. For fixed $\Lambda, \lambda$ displays a maximum at a temperature slightly higher than $\epsilon$ in the TLS model, as shown in Figure 8 (right panel). In Figure 9, the fwhm (full-width at half-maximum) of $\sigma(\omega)$ is plotted versus temperature $T$. For the $\mathrm{BO}$ model, the line width $\sim \sqrt{\lambda k_{\mathrm{B}} T}$ grows with the temperature, while for the TLS model, it approaches a finite 

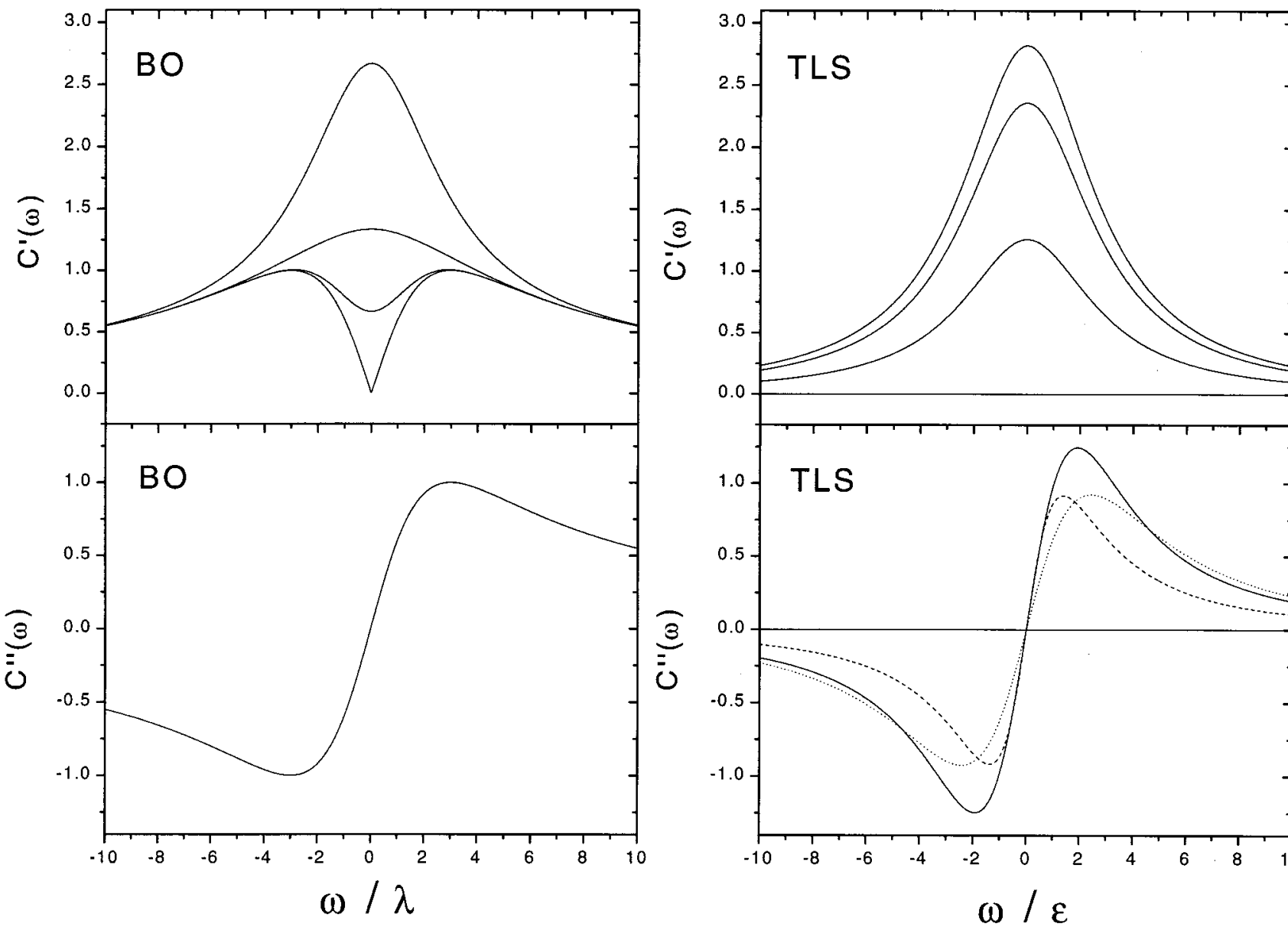

Figure 6. Comparison of the Brownian oscillator model (left panel) and the TLS model (right panel): temperature dependence of $C^{\prime}(\omega)$ and $C^{\prime \prime}(\omega) . C^{\prime}(\omega)$ in both models increases with temperature: from top down, $T=2,1,0.5,0$. In the Brownian oscillator model $\lambda$ is used as the energy unit, while in the TLS model $\epsilon$ is used as the energy unit. $\Lambda=3$. $C^{\prime \prime}(\omega)$ for the Brownian oscillator model has no temperature dependence (left, lower panel). $C^{\prime \prime}(\omega)$ for the TLS model (right, lower panel) peaks at $T=\epsilon$ (solid). Also plotted in that panel are $T=0.5 \epsilon($ dashed) and $T=2 \epsilon$ (dotted).

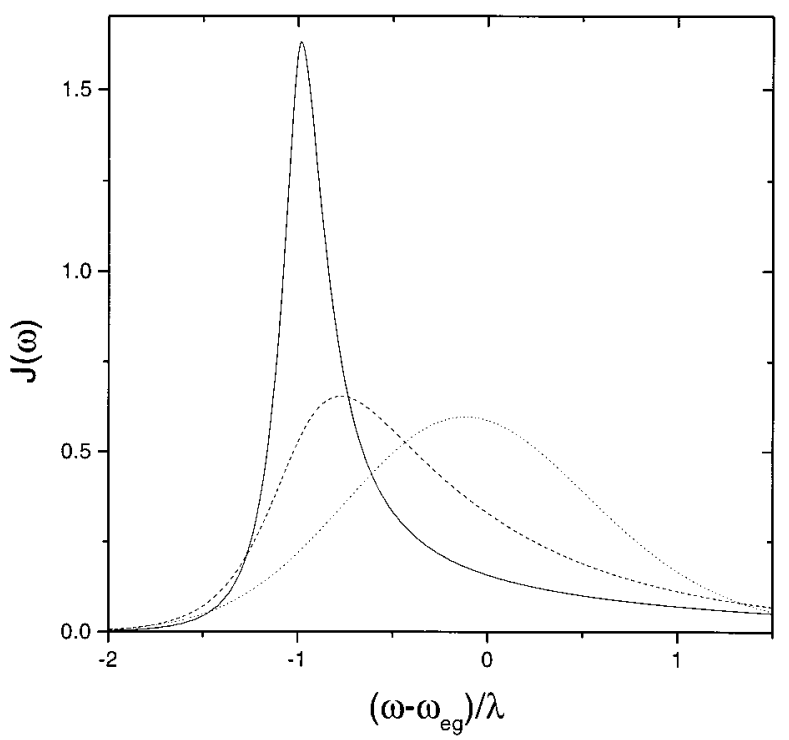

Figure 7. Absorption line shapes of the overdamped Brownian oscillator model. $\lambda$ is used as the energy unit. $T=0.25 \lambda$. Three values of $\Lambda / \lambda$ are shown: 3 (solid), 1 (dashed), and 0.3 (dotted).

limiting value at high temperatures. The temperature dependence reflects the difference between BO and TLS asymptotics. At high temperatures, the boson bath becomes Gaussian while the spin bath saturates. The TLS model is usually applied at low temperatures, where the two baths show similar behaviors.
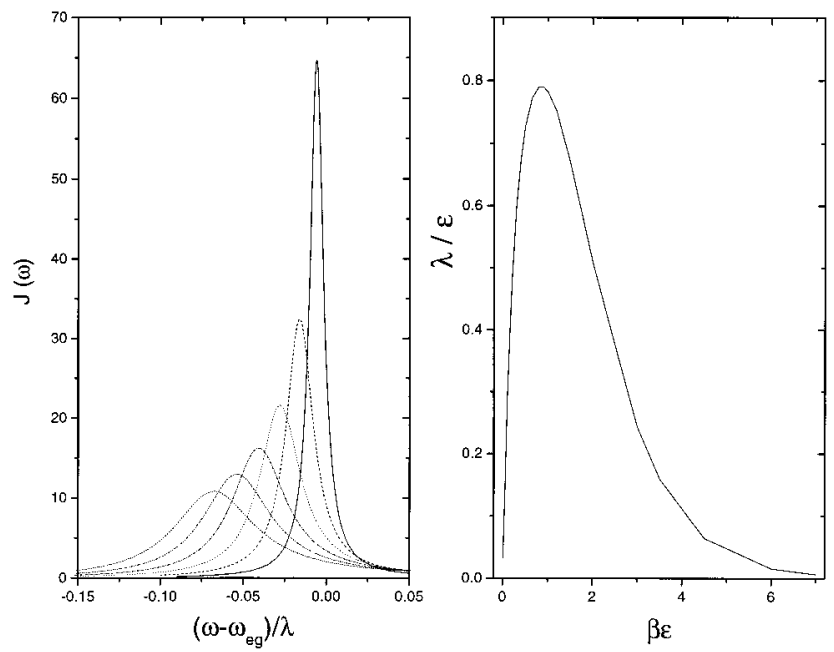

Figure 8. Absorption line shapes of the extended TLS model. $T=$ $\epsilon / 6$. Six values of $\Lambda / \epsilon$ are shown (left panel), from left to right: 6,5 , $4,3,2,1$. For a fixed rate $\Lambda=\epsilon, \lambda$ is plotted as a function of $\beta$ (right panel). A maximum is found at a temperature slightly higher than $\epsilon$.

It should be noted that the fluctuation-dissipation theorem, which has been used in deriving eq 5.1, fixes the ratio $C^{\prime}(\omega) /$ $C^{\prime \prime}(\omega)$ to be $\operatorname{coth}(\beta \hbar \omega / 2)$, but does not specify the temperature dependence of $C^{\prime}(\omega)$ and $C^{\prime \prime}(\omega)$. For the microscopic Brownian oscillator model $C^{\prime \prime}(\omega)$ is temperature independent. We show that in the weak coupling limit when the TLS model resembles 

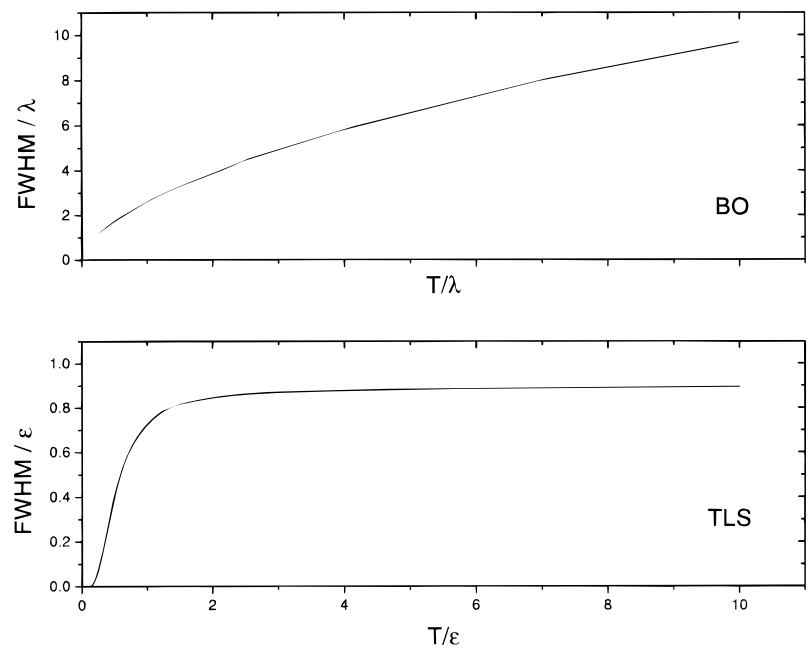

Figure 9. Full-width at half-maxima (fwhm) of the absorption line shapes as a function of temperature for the two models: BO model (upper panel, $\Lambda=\lambda$ ); TLS model (lower panel, $\Lambda=\epsilon$ ).

the oscillator model, the line-shape function has the form similar to that of the Brownian oscillator model with the only difference that $C^{\prime}(\omega)$ rather than $C^{\prime \prime}(\omega)$ is temperature independent. This is the motivation for representing $g^{\mathrm{TLS}}(t)$ in the form of eq 5.1 rather than eq 5.3 .

\section{Multipolar Coupling to a Spin Bath}

Assuming a general multipolar chromophore-TLS coupling in $d$ dimesions,

$$
v_{j} \propto r_{j}^{-n}
$$

the distribution function $n(k, v, E)$ takes the form

$$
n(k, v, E) \propto v^{-(1+d / n)} \delta(k-K) \delta\left(E-E_{0}\right)
$$

For dipolar interations,

$$
v_{j}=c r_{j}^{-3} f\left(\theta_{j}\right)
$$

where $\mathbf{r}_{j}$ points from the chromophore to the $j$ th TLS, $\theta_{j}$ is the angle between the $z$-axis and $\mathbf{r}_{j}$. The dipolar chromophoreTLS coupling yields a distribution function $n(k, v, E)$

$$
n(k, v, E) \propto \frac{1}{v^{2}} \delta(k-K) \delta\left(E-E_{0}\right)
$$

where the proportionality prefactor depends on the angular form of the chromophore-TLS coupling. The dipolar chromophoreTLS coupling of eq 6.3 is believed to be mediated by accoustic phonons. ${ }^{17,22,59,60}$

The parameters for the chromophore-TLS system include $v, k, E, \rho, a, R_{\max }$, and $T$, where $E$ is the energy separation of TLS, $\rho$ is the TLS density, $a$ represents the distance between the chromophore and the closest TLS (which is the same order of magnitude as the lattice constant), and $R_{\max }$ is the largest possible separation between the chromophore a TLS (which roughly stands for the physical size of the system).

To clarify the applicable range of the central limit (cumulant) expansion, we note that TLSs can be generally classified into either "distant" or "nearby" systems according to their distance from the chromophore. Nearby systems give large but infrequent modulations, whereas distant systems give small but frequent modulation. For nonstatic systems, there exists a natural criterion of this classification: whether coupling is larger or smaller than the rate of TLS flipping, For static systems, however, a small sphere of radius $R_{c}$ is usually carved out around the chromphore to define the remaining TLS as distant. The central limit theorem ${ }^{61}$ is only applicable to the distant systems at long times.

We note that, for example, for parallel dipoles $f(\theta)=1-\cos ^{2} \theta$,

$$
\int \mathrm{d} \Omega f(\theta)=0
$$

That is, the chromophore-TLS coupling has equal probability to be attractive or repulsive. This being assumed, the configurational average then takes the form

$$
\langle\rangle_{c}=\frac{1}{V} \int_{0}^{\infty} r^{2} \mathrm{~d} r \int \mathrm{d} \Omega
$$

For dipolar coupling, we examine a static system $(k=0)$ at low temperatures $(p \rightarrow 0)$. We recast the first term in eq G1 with

$$
g_{c}(t)=\left\langle p\left(1-\mathrm{e}^{-\mathrm{i} v t}\right)\right\rangle_{c}
$$

For any real constant $C$, we have

$$
\int_{0}^{\infty} x^{2} \mathrm{~d} x\left[1-\cos \left(\frac{C}{x^{3}}\right)\right]=\frac{\pi}{6}|C|
$$

Carrying out the intergrations over $r$ in the configurational average given by eq 6.6 , we obtain

$$
g_{c}(t)=\Gamma|t|
$$

where $\Gamma$ is proportional to $p$ and depends on the detailed form of chromophore-TLS coupling $f(\theta)$ :

$$
\Gamma=\frac{\pi c \rho p}{6} \int \mathrm{d} \Omega\left|1-3 \cos ^{2} \theta\right|=\frac{8 \pi^{2}}{9 \sqrt{3}} c \rho p
$$

Therefore the line-shape function is a Lorentzian. This result was first derived in the seminal paper of Stoneham. ${ }^{62}$ In order to obtain the Lorentzian line shape, it is essential to set the lower cutoff $a$ to zero.

At high temperatures $(\kappa \ll 1, T \rightarrow \infty)$, the rates for up and down transitions are equal (for simplicity, we ignore the variation in the rates):

$$
k_{j}^{d}=k_{j}^{u}=\frac{K_{j}}{2}=\frac{K}{2}
$$

The TLS can be divided into nearby $\left(v_{j}^{2}>K_{j}^{2}\right)$ and distant $\left(v_{j}^{2}<K_{j}^{2}\right)$ systems. After the configurational average, the bulk response function follows from eq 4.2 and eq 6.6:

$$
\frac{1}{N} g_{c}(t)=A\left[f_{1}\left(\frac{K t}{2}\right)+f_{2}\left(\frac{K t}{2}\right)\right]
$$

where the constant $A$ depends on the angular distribution of $v_{j}$. The nearby systems contribute

$f_{1}(y)=$
$\int_{0}^{\infty} x\left(1+x^{2}\right)^{-3 / 2}\left[1-\mathrm{e}^{-y} \cos (x y)-\mathrm{e}^{-y} x^{-1} \sin (x y)\right] \mathrm{d} x$

and the distant systems contribute 


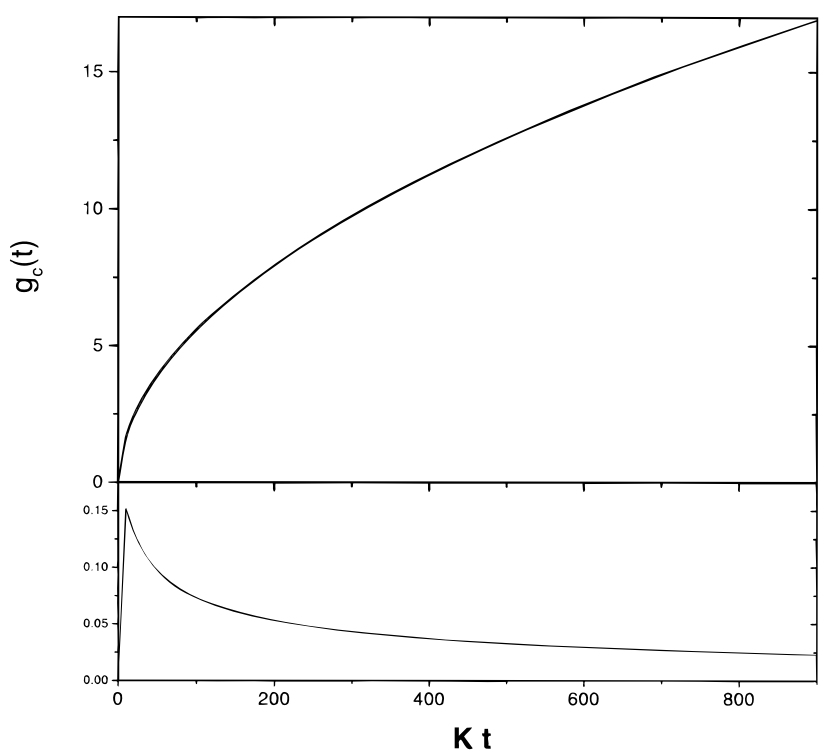

Figure 10. Bulk response function $g_{c}(t)$ and its Padé approximant (upper panel). The curves shown are without the prefactor $(\pi / 2)^{2} N A$. The difference of the two curves is displayed in the lower panel.

$f_{2}(y)=$

$\int_{0}^{1} x\left(1-x^{2}\right)^{-3 / 2}\left[1-\mathrm{e}^{-y} \cos (x y)-\mathrm{e}^{-y} x^{-1} \sin (x y)\right] \mathrm{d} x$

Combining the two contributions,

$$
\frac{1}{N} g_{c}(t)=\frac{\pi A K t}{4} \mathrm{e}^{-K t / 2}\left[I_{0}\left(\frac{K t}{2}\right)+I_{1}\left(\frac{K t}{2}\right)\right]
$$

where $I_{0}(x)$ and $I_{1}(x)$ are modified Bessel functions of zeroth and first order. We have constructed a Padé approximant for $g_{c}(t)$ :

$$
\frac{2}{\pi} \frac{1}{N^{3}} g_{c}(t) \approx \frac{\pi}{2} A \frac{\frac{K t}{2}+\sqrt{\frac{2}{\pi}}\left(\frac{K t}{2}\right)^{3 / 2}}{1+1.243\left(\frac{K t}{2}\right)^{1 / 2}+\frac{K t}{2}}
$$

In Figure 10 we compare eqs 6.15 and 6.16. The two curves are very close over the entire time range.

We point out two limiting cases of eq 6.12 assuming $f(\theta)=1-3 \cos ^{2} \theta$. For the static limit $K t \ll 1$, TLS either does not flip or at most flips once; one has

$$
\bar{J}(t)=\exp \left[-\frac{\pi}{12} \bar{c} \rho t\left(1-\frac{K t}{4}\right)\right]
$$

with

$$
\bar{c}=c \int \mathrm{d} \Omega\left|1-\cos ^{2} \theta\right|=\frac{16 \pi c}{3 \sqrt{3}}
$$

and the line shape is Lorentzian. In the opposite limit $K t \gg 1$,

$$
\bar{J}(t)=\exp \left[-\frac{\pi}{12}\left(\frac{2}{\pi}\right)^{1 / 2} \bar{c} \rho\left(\frac{2 t}{K}\right)^{1 / 2}\right]
$$

The line shapes obtained using eqs $6.9,6.17$, and 6.19 have the form of Levy distributions. ${ }^{63,64,71}$

Finally, the line-shape function $\sigma(\omega)$ for the dipolar coupling case is the one-sided Fourier transform of the response function $J_{c}(t) . \sigma(\omega)$ is quite sensitive to the TLS density $\rho$. The smaller the density, the sharper the line shape. In Figure 11, we display

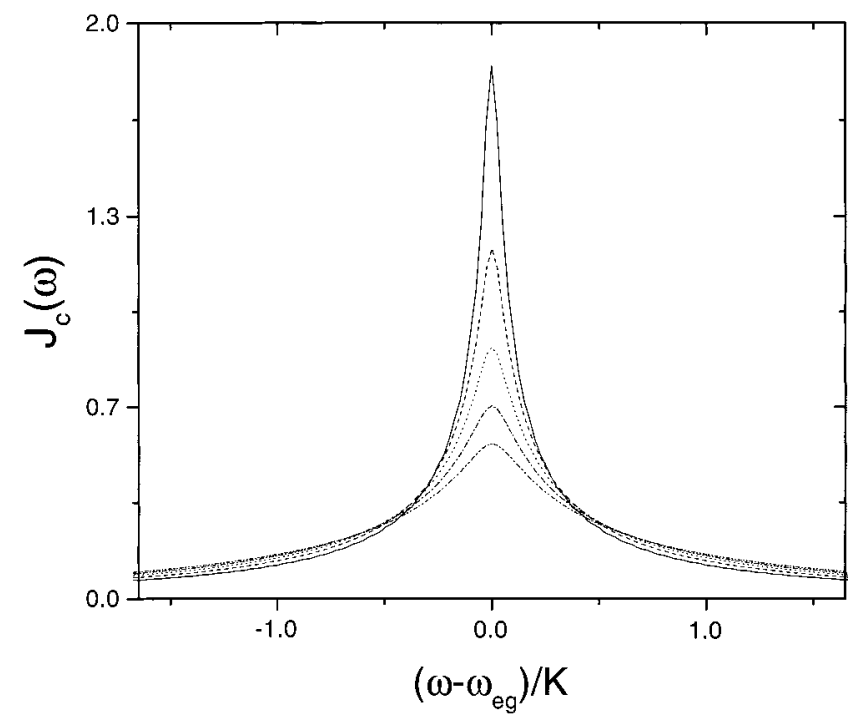

Figure 11. Line-shape function $J_{c}(\omega)$ for dipolar coupling is shown for five values of $z=\pi N A / K$ eq 6.20 which is proportional to the TLS density $\rho$ : from top down, $z=1.1,1.4,1.7,2.0,2.3$.

$\sigma(\omega)$ for five values of $z(z=1.1,1.4,1.7,2.0,2.3)$, where $z$ is proportional to the TLS density:

$$
z=\frac{\pi N A}{K} \propto \frac{\rho}{K}
$$

We note that for a fixed TLS density $\rho$, the slower the bath, the closer the line shape is to a Lorentzian. This is consistent with our discussion of the static limit.

For bulk measurements of chromophores in a glassy matrix, the nonlinear response functions have to be averaged over the TLS configurations. Neglecting variations in up and down rates of TLS, i.e., $K_{j}=K$, the configurationally averaged three-pulseecho response function takes the form ${ }^{37-40}$

$$
\bar{R}_{2}\left(t_{1}, t_{2}, t_{1}\right)=\exp \left\{-\rho\left[2 K \int_{0}^{t_{1}} \mathrm{~d} t \bar{F}(t)+\left(1-\mathrm{e}^{-K t_{2}}\right) \bar{F}\left(t_{1}\right)\right]\right\}
$$

where $t_{2}$ and $t_{1}$ separate the first pulse from the second, and the second from the third, respectively. The auxiliary function $\bar{F}(t)$ is defined as

$$
\bar{F}(t)=\frac{\pi \bar{c} k^{d} k^{u}}{3 K^{2}} \mathrm{e}^{-K t} \int_{0}^{t} \mathrm{~d} t^{\prime} I_{0}\left(K t^{\prime}\right) I_{0}\left[K\left(t-t^{\prime}\right) \tanh \left(\frac{\beta E}{2}\right)\right]
$$

\section{Discussion}

In this paper we have compared the Brownian oscillator (boson bath) and the TLS (spin bath) models for the optical responses of chromophores in the condensed phase. For the Brownian oscillator model we can relate the spectral density functions to the third-order response functions via eq 2.14 using the second-order cumulant expansions. This relation is exact for the boson bath. In order to verify to what degree such relations between the linear response and third-order response are retained in the TLS model, we expanded the linear and thirdorder response functions to the fourth power in the chromophore-TLS coupling strength. We have found that to second order in the chromophore-TLS coupling strength eq 2.14 holds for the TLS model. At higher orders, however, deviations from eq 2.14 have been found.

The TLS model assumes that the bath modulates the transition frequency of the chromophore. However, the model fails to 
take into account that the optical excitation of the chromophore can alter the surrounding TLS via the chromophore-TLS coupling. Relaxation of the TLS that follows will add to the equilibrium thermal fluctuations of the TLS. In order to describe this effect, we extend the TLS model to include the imaginary part of the spectral density function by means of the fluctuationdissipation theorem. Absorption line-shape functions were calculated and compared with the Brownian oscillator model. A very different temperature dependence of the absorption line shape has been found for the two cases.

A microscopic model of phonon-mediated chromophoreTLS coupling including TLS dynamics was proposed by Suárez and Silbey ${ }^{59}$ in order to provide insight into the range of validity of the stochastic TLS model. Path-integral techniques show that each stochastic trajectory corresponds to a series of quautum paths. It was concluded that the stochastic TLS model can be derived from a microscopic Hamiltonian if the TLS dynamics are uncorrelated.

To gain further insight into the stochastic TLS model, we also examined a microscopic model for a spin bath in Appendix H. Cumulant expansions are performed for the spectral broadening function. To second-order in chromophorebath coupling strength, the model reduces to the Brownian oscillator model. This points to the universal nature of the Brownian oscillator model in the weak coupling regime of hostbath systems. The slow modulation and high-temperature limits of the TLS model are discussed in Appendix G.

The relations between the boson and spin baths can be visualized using microscopic models. For the boson bath, the chromophore is linearly coupled to a set of harmonic oscillators in the microscopic model. If there are $N$ identical oscillators linearly coupled to the chromophore, one can find a linear canonical transformation resulting in $N$ transformed harmonic oscillators, one of which is coupled the chromophore, and the rest, $N-1$, are uncoupled. This implies that for a given bath frequency the bath is represented by a single effective oscillator. This rationalizes the well-established description of a boson bath using a collective coordinate and a spectral density that represents the correlation functions of the collective coordinate. ${ }^{3,65}$ The collective coordinate is given by the sum over all bath frequencies of the coupled effective oscillators (one per frequency), whereas the spectral density is represented by the square of the coupling between the effective oscillator of frequency $\omega$ and the chromophore. This picture is, however, limited to harmonic systems only, and therefore, does not apply to the spin bath, which is highly anharmonic. This is why for a spin bath we need more detailed information about the bath, namely, all individual coupling constants. For the microscopic model all the relevant information is contained in the distribution function of the frequencies and coupling constants $n(\omega, u)$ introduced in Appendix $\mathrm{H}$, whereas the stochastic model can be described by the distribution function $n(k, v, E)$ introduced in Section VI. Adopting the language of correlation functions, a harmonic system can be described by a two-point correlation function related to the spectral density since all higher-order ones can be expressed in terms of the two-point correlation functions using Wick's theorem. For an anharmonic (e.g., spin) bath, the optical response involves high-order correlation functions and may not be represented in terms of a single spectral density function.

We have shown, however, that in the case of a large number $(N)$ of TLSs weakly coupled to the chromophore with coupling strength $v$, the optical response reproduces that of the boson bath in the limit of $N \rightarrow \infty$ and $v^{2} N=$ const. This result has a clear interpretation: $N$ spins of $S=1 / 2$ can be naturally represented as a collection of spins from 0 to $N / 2$ (if $N$ is even). Since large $S$ spins behave almost as bosons (see, e.g., ref 66), the behavior of the boson system is reproduced.

The similarity between the fermion and boson baths has been explored through real-time finite-temperature dynamics. ${ }^{67}$ It was concluded that for ohmic disspation (i.e., $\gamma_{j}(\omega)=$ const) the two baths have a similar behavior. Additional evidence is provided by an approximate mapping of the anisotropic Kondo model $^{68}$ onto the spin-boson Hamiltonian with ohmic dissipation using bosonization techniques, ${ }^{69,70}$ where the oscillators in the spin-boson model play the role of the spin-denisty excitations in the Kondo case. Since $S=1 / 2$ spins correspond to spinless fermions, it is not surprising that spin and boson baths share common characteristics. This can also be applied to a bath of spins of size $S$ because fermion statistics is gradually replaced by boson statistics with increasing $S$.

In this paper, the connection between the two baths has been established for a much more general situation: all one needs is to have the TLS distribution function $n(k, v, E)$ be concentrated in the $v \ll k$ regime in order for the spin bath to behave as the boson bath. The oscillator limit and the deviations are obtained using a straightforward cumulant expansion in powers of $v / k$ carried out in Section VI. The static limit for the TLS model is defined by the condition $k \ll v$. Nontrivial line shapes for the TLS model occur when $n(k, v, E)$ is concentrated in neither $k \ll v$ or $k \gg v$ regimes.

Apart from the obvious $k \ll v$ (static) limit, there is another static limit where the TLS model reproduces the boson model in the static limit. This occurs when $N p(1-p) v^{2} / k \gg k$ (which can hold even when $v \ll k$ provided $N$ is sufficiently large). In this case the line shape is Gaussian, as is known in the static limit of the boson model. Using the TLS model, the static limit of the boson model has the following meaning: although the TLS is formally in the fast limit $(k \gg v)$ and the TLS flippings occur frequently, the changes of the total spin comparable to N/2 occur very rarely, which means that the effective boson system is in the static limit. In particular, this provides a clear interpretation of the dependence of the line shape for the multipolar model of Section VI on the regulization parameter $a$ in the static limit. If $a$ is small, the dominant contribution comes from the $k \ll v$ regime, and one can set $k=0$, which yields the result of ref 62 with the Lorentzian line shape (eq 6.17). In the opposite limit of large $a, v \ll k$ for all TLSs, and the static limit of the boson model yields a Gaussian line shape. This implies that depending on the value of $a$ the line shapes in the static limit $k \rightarrow 0$ interpolate between the Lorentzian and the Gaussian form.

The first successful realization of single molecule spectroscopy (SMS) at low temperatures has been performed on pentacene chromophores in $p$-terphenyl crystals. SMS allows us to probe the immediate microscopic environment of a single molecule, usually in tens of nanometers, eliminating the normal ensemble averages over the bulk material required by the classical methods. In the context of single-molecule spectroscopy, the role of disorder clearly comes into play as each chromophore is uniquely positioned with respect to the TLS. Numerical work by Zumofen and Klafter ${ }^{71}$ reveals that for a large but finite number of TLSs ordered and disordered systems have similar behaviors at short times, but at long times, TLS on a filled lattice induce a Gaussian line shape while disorder TLSs give line shapes depending on particular realizations. It was conjectured that for disordered TLSs line shapes after averaging over realizations would follow a Lorentzian. It will 
be therefore interesting to see how line shapes change from Gaussian to a Lorentzian form upon the onset of disorder which can be simply introduced by allowing ordered TLSs to displace from lattice points. Similarity can be drawn between this case and the problem of hysteresis in a magnetic particle system where positional disorder of point dipoles is believed to be responsible for hysteretical behavior of the system. ${ }^{72}$ It is also interesting to compare numerical models of finite numbers of TLSs and models of infinte spins ${ }^{73}$ and continuum models of a spin bath. For example, the continuum model gives a Lorentzian in the static limit, while a filled lattice of TLS is found numerically to give a Gaussian line shape. For this case, the difference lies in the lower cutoff $a$, which tends to zero in the continuum model but not in a lattice model.

Acknowledgment. The support of the National Science Foundation (Grants No. PHY94-15583 and CHE95-26125) and U.S. Air Force Office of Scientific Research (Grant No. F4962096-1-0030) is gratefully acknowledged.

\section{Appendix A: Brownian Oscillator (Spin-Boson) Model}

In this Appendix we derive an expression for the spectral density of a boson bath. In the Brownian oscillator model, the system is taken to be a two-electronic-level system with some primary nuclear coordinates coupled linearly to the electronic systems:

$$
\hat{H}^{\mathrm{BO}}=|g\rangle \hat{H}_{g}^{\mathrm{BO}}\langle g|+| e\rangle \hat{H}_{e}^{\mathrm{BO}}\langle e|+\hat{H}^{\prime}
$$

where

$$
\begin{gathered}
\hat{H}_{g}^{\mathrm{BO}}=\sum_{j}\left[\frac{p_{j}^{2}}{2 m_{j}}+\frac{1}{2} m_{j} \omega_{j}^{2} q_{j}^{2}\right] \\
\hat{H}_{e}^{\mathrm{BO}}=h \omega_{e g}^{0}+\sum_{j}\left[\frac{p_{j}^{2}}{2 m_{j}}+\frac{1}{2} m_{j} \omega j^{2}\left(q_{j}+d_{j}\right)^{2}\right] \\
\hat{H}^{\prime}=\sum_{n}\left[\frac{p_{n}^{2}}{2 m_{n}}+\frac{m_{n} \omega_{n}^{2}}{2}\left(x_{n}-\sum_{j} \frac{c_{n j} q_{j}}{m_{n} \omega_{n}{ }^{2}}\right)^{2}\right]
\end{gathered}
$$

Here $p_{j}\left(p_{n}\right), q_{j}\left(x_{n}\right)$, and $m_{j}\left(m_{n}\right)$ represent the momentum, the coordinate, and the mass of the $j$ th $(n$ th) nuclear mode of the primary (bath) oscillators, respectively. $H^{\prime}$ describes the bath oscillators and their coupling to the primary oscillators with a coupling strength $c_{n}$. For this model, the second-order cumulant expansions 2.14 are exact, and we have

$$
C^{\prime \prime}(\omega)=\sum_{j} C_{j}^{\prime}(\omega)
$$

where

$$
C_{j}^{\prime}(\omega)=\left(\frac{m_{j} \omega_{j}^{4} d_{j}^{2}}{2 \hbar}\right) \frac{\omega \gamma_{j}(\omega)}{\left(\omega_{j}^{2}+\omega \Sigma_{j}(\omega)-\omega^{2}\right)^{2}+\omega^{2} \gamma_{j}^{2}(\omega)}
$$

Here $\gamma_{j}(\omega)$ represents the spectral distribution of the coupling

$$
\gamma_{j}(\omega)=\frac{\pi}{m_{j}} \sum_{n} \frac{c_{n j}{ }^{2}}{2 m_{n} \omega_{n}{ }^{2}}\left[\delta\left(\omega-\omega_{n}\right)+\delta\left(\omega+\omega_{n}\right)\right]
$$

and the real part $\Sigma_{j}(\omega)$ of the self-energy is related to the spectral distribution of the coupling by the Kramers-Kronig relation ${ }^{56}$

$$
\Sigma_{j}(\omega)=-\frac{1}{\pi} \operatorname{Re} \int_{-\infty}^{\infty} \mathrm{d} \omega^{\prime} \frac{\gamma_{j}\left(\omega^{\prime}\right)}{\omega^{\prime}-\omega}
$$

This gives

$$
\sum_{j}(\omega)=\frac{1}{m_{j}} \operatorname{Re} \sum_{n} \frac{c_{n j}^{2}}{2 m_{n} \omega_{n}^{2}}\left(\frac{1}{\omega-\omega_{n}+\mathrm{i} \eta}+\frac{1}{\omega+\omega_{n}+\mathrm{i} \eta}\right)
$$

\section{Appendix B: Linear Response of the Two-State Jump Model}

In this Appendix we derive a closed expression for the linear response function of the stochastic TLS model. The response function $S^{(1)}(t)$ can be calculated using the Green function approach. ${ }^{3}$ The response functin $J(t)$ can be written as

$$
J(t)=\left\langle\left\langle V_{e g}\left|G_{e g}(t)\right| V_{e g} \rho_{g}\right\rangle\right\rangle
$$

Here $\rho_{g}$ is the equilibrium density operator, and the Green function $\mathrm{G}_{m n}(t)$ ( $m, n$ can be $e$ or $g$ ) is defined by its action on operator $\hat{A}$ for a time-dependent Hamiltonian as

$$
\mathrm{G}_{m n}(t) \hat{A} \equiv \theta(t) \exp \left[-\frac{\mathrm{i}}{\hbar} \int_{0}^{t} \mathrm{~d} \tau H_{n}(\tau)\right] \hat{A} \exp \left[\frac{\mathrm{i}}{\hbar} \int_{0}^{t} \mathrm{~d} \tau H_{m}(\tau)\right]
$$

$J(t)$ takes the following form if the identity matrices are inserted in the above equation of $J(t)$ :

$$
J(t)=\langle\langle V \mid e g\rangle\rangle\left\langle\left\langle e g\left|\mathrm{G}_{e g}(t)\right| e g\right\rangle\right\rangle\langle\langle e g|\mathrm{~V}| g g\rangle\rangle\langle\langle g g \mid \rho(-\infty)\rangle\rangle
$$

The TLS populations satisfy ordinary rate equations as given by eq 3.2. Each Liouville space vector is now a two-component column vector in the TLS population space,

$$
\begin{gathered}
\langle\langle V \mid e g\rangle\rangle=\mu\left(\begin{array}{ll}
1 & 1
\end{array}\right) \\
\langle\langle g g \mid \rho(-\infty)\rangle\rangle=\left(\begin{array}{l}
1-p_{j} \\
p_{j}
\end{array}\right)
\end{gathered}
$$

Liouville space operators such as $\mathrm{V}$ and $\mathrm{G}_{e g}$ become $2 \times 2$ matrices in the same space,

$$
\langle\langle e g|\mathrm{~V}| g g\rangle\rangle=\mu\left(\begin{array}{ll}
1 & 0 \\
0 & 1
\end{array}\right)
$$

The Laplace transform of the Green function $\mathrm{G}_{e g}(t)$ is

$$
\left\langle\left\langle e g\left|\tilde{\mathrm{G}}_{e g}(s)\right| e g\right\rangle\right\rangle=\left(\begin{array}{ll}
s+\mathrm{i} \omega_{e g}+k_{j}^{u} & -k_{j}^{d} \\
-k_{j}^{u} & s+\mathrm{i} \omega_{e g}+\mathrm{i} v_{j}+k_{j}^{d}
\end{array}\right)^{-1}
$$


The time-domain response function due to the $j$ th TLS,

$$
J_{j}^{a}(t)=\mathrm{e}^{-\mathrm{i} \omega_{0} t} J_{j}(t)
$$

can be easily obtained by an inverse Laplace transform. Alternatively, we introduce the one-sided Fourier transform ${ }^{3}$

$$
\begin{gathered}
\theta(t) f(t)=-\frac{1}{2 \pi \mathrm{i}} \int_{-\infty}^{\infty} \mathrm{d} \omega \tilde{F}_{j}(\omega) \mathrm{e}^{-\mathrm{i} \omega t} \\
\tilde{F}_{j}(\omega)=-\mathrm{i} \int_{0}^{\infty} \mathrm{d} t f(t) \mathrm{e}^{\mathrm{i} \omega t}
\end{gathered}
$$

which appears frequently in linear and nonlinear spectra calculations. In the frequency domain, one obtains

$$
\tilde{J}_{j}(\omega)=\frac{\left(\omega-\omega_{e g}\right)-\mathrm{i} K_{j}-v_{j}\left(1-p_{j}\right)}{\left(\omega-\omega_{e g}\right)\left(\omega-\omega_{e g}-\mathrm{i} K_{j}\right)-v_{j}\left(\omega-\omega_{e g}-\mathrm{i} K_{j} p_{j}\right)}
$$

$J_{j}^{a}(t)$ (eq B8) is the one-sided Fourier transform of the response function $\tilde{J}_{j}(\omega)$.

\section{Appendix C: Third-Order Response in the Frequency Domain}

In this Appendix we derive expressions for the frequency domain third-order nonlinear optical response of the TLS model. In the frequency domain, we have

$$
\begin{aligned}
& S^{(3)}\left(\omega_{1}+\omega_{2}+\omega_{3}, \omega_{1}+\omega_{2}, \omega_{1}\right)= \\
& \left(-\frac{1}{\hbar}\right)^{3} \sum_{\alpha=1}^{4}\left[R_{\alpha}\left(\omega_{1}+\omega_{2}+\omega_{3}, \omega_{1}+\omega_{2}, \omega_{1}\right)+\right. \\
& \left.R_{\alpha}^{*}\left(-\omega_{1}-\omega_{2}-\omega_{3},-\omega_{1}-\omega_{2},-\omega_{1}\right)\right](\mathrm{C} 1
\end{aligned}
$$

where

$$
\begin{aligned}
& R_{\alpha}\left(\omega_{1}+\omega_{2}+\omega_{3}, \omega_{1}+\omega_{2}, \omega_{1}\right) \equiv \\
& (-\mathrm{i})^{3} \int_{0}^{\infty} \mathrm{d} t_{3} \int_{0}^{\infty} \mathrm{d} t_{2} \int_{0}^{\infty} \mathrm{d} t_{1} R_{\alpha}\left(t_{3}, t_{2}, t_{1}\right) \exp \left[\mathrm{i}\left(\omega_{1}+\omega_{2}+\omega_{3}\right) t_{3}+\right. \\
& \left.\mathrm{i}\left(\omega_{1}+\omega_{2}\right) t_{2}+\mathrm{i} \omega_{1} t_{1}\right]
\end{aligned}
$$

We first examine one of the response functions in the frequency domain

$$
\begin{aligned}
& R_{1}\left(\omega_{1}+\omega_{2}+\omega_{3}, \omega_{1}+\omega_{2}, \omega_{1}\right)= \\
& \quad\left(\begin{array}{ll}
1 & 1
\end{array}\right) G_{e g}\left(\omega_{1}+\omega_{2}+\omega_{3}\right) G_{e e}\left(\omega_{1}+\omega_{2}\right) G_{e g}\left(\omega_{1}\right)\left(\begin{array}{c}
\rho_{e q}^{e} \\
\rho_{e q}^{e^{\prime}}
\end{array}\right)
\end{aligned}
$$

where

$$
\left(\begin{array}{l}
\rho_{e q}^{e} \\
\rho_{e q}^{e^{\prime}}
\end{array}\right)=\left(\begin{array}{l}
1-p_{j} \\
p_{j}
\end{array}\right)
$$

and $G_{e g}(\omega)$ and $G_{e e}(\omega)$ are the Green functions.
$R_{1}\left(\omega_{1}+\omega_{2}+\omega_{3}, \omega_{1}+\omega_{2}, \omega_{1}\right)$ can be simplified as (cf. Appendix A)

$$
\begin{aligned}
& R_{1}\left(\omega_{1}+\omega_{2}+\omega_{3}, \omega_{1}+\omega_{2}, \omega_{1}\right)= \frac{1}{f\left(\omega_{1}, \omega_{2}, \omega_{3}\right)} \times \\
&\left(\omega_{1}+\omega_{2}+\omega_{3}-\omega_{e g}-v_{j}+\mathrm{i} k_{p}, \omega_{1}+\omega_{2}+\right. \\
&\left.\omega_{3}-\omega_{e g}-\mathrm{i} k_{p}\right)\left(\begin{array}{ll}
\omega_{1}+\omega_{2}+\mathrm{i} \frac{k^{\prime}}{2} & -\mathrm{i} \frac{k^{\prime}}{2} \\
-\mathrm{i} \frac{k}{2} & \omega_{1}+\omega_{2}+\mathrm{i} \frac{k}{2}
\end{array}\right) \times \\
&\left(\begin{array}{l}
\left(1-p_{j}\right)\left(\omega_{1}-\omega_{e g}-v_{j}+\mathrm{i} k_{p}\right) \\
p_{j}\left(\omega_{1}-\omega_{e g}-\mathrm{i} k_{p}\right)
\end{array}\right)
\end{aligned}
$$

where we have introduced the parameter

$$
k_{p}=\left(1-2 p_{j}\right) K_{j}
$$

which vanishes at high temperatures, and

$$
\begin{array}{r}
f^{\prime}\left(\omega_{1}, \omega_{2}, \omega_{3}\right)= \\
\left(\omega_{1}+\omega_{2}+\omega_{3}-\mathrm{i} \Omega_{+}\right)\left(\omega_{1}+\omega_{2}+\omega_{3}-\mathrm{i} \Omega_{-}\right) \times \\
\left(\omega_{1}+\omega_{2}\right)\left(\omega_{1}+\omega_{2}+\mathrm{i} K\right)\left(\omega_{1}-\mathrm{i} \Omega_{+}\right)\left(\omega_{1}-\mathrm{i} \Omega_{-}\right)
\end{array}
$$

with

$$
\begin{gathered}
\Omega_{+}=\Omega_{j}+\frac{b_{j}}{2} \\
\Omega_{-}=-\Omega_{j}+\frac{b_{j}}{2}
\end{gathered}
$$

Similarly, in the frequency domain,

$$
\begin{gathered}
R_{2}\left(\omega_{1}+\omega_{2}+\omega_{3}, \omega_{1}+\omega_{2}, \omega_{1}\right)=\frac{1}{f\left(\omega_{1}, \omega_{2}, \omega_{3}\right)} \times \\
\left(\omega_{1}+\omega_{2}+\omega_{3}-\omega_{e g}-v_{j}+\mathrm{i} k_{p}, \omega_{1}+\omega_{2}+\right. \\
\left.\omega_{3}-\omega_{e g}-\mathrm{i} k_{p}\right)\left(\begin{array}{ll}
\omega_{1}+\omega_{2}+\mathrm{i} \frac{k^{\prime}}{2}-\mathrm{i} \frac{k^{\prime}}{2} \\
-\mathrm{i} \frac{k}{2} & \omega_{1}+\omega_{2}+\mathrm{i} \frac{k}{2}
\end{array}\right) \times \\
\left(\begin{array}{l}
\left(1-p_{j}\right)\left(\omega_{1}+\omega_{e g}+v_{j}-\mathrm{i} k_{p}\right) \\
p\left(\omega_{1}+\omega_{e g}+\mathrm{i} k_{p}\right)
\end{array}\right)
\end{gathered}
$$

where

$$
\begin{aligned}
& f^{\prime}\left(\omega_{1}, \omega_{2}, \omega_{3}\right)= \\
&\left(\omega_{1}+\omega_{2}+\right.\left.\omega_{3}-\mathrm{i} \Omega_{+}\right)\left(\omega_{1}+\omega_{2}+\omega_{3}-\mathrm{i} \Omega_{-}\right)\left(\omega_{1}+\omega_{2}\right) \\
&\left(\omega_{1}+\omega_{2}+\mathrm{i} K\right)\left(\omega_{1}+\mathrm{i} \Omega_{+}\right)\left(\omega_{1}+\mathrm{i} \Omega_{-}\right)(\mathrm{C} 10)
\end{aligned}
$$


Substituting the three Green functions $G_{e g}\left(\omega_{1}+\omega_{2}+\omega_{3}\right)$, $\mathrm{G}_{e e}\left(\omega_{1}+\omega_{2}\right)$, and $\mathrm{G}_{e g}\left(\omega_{1}\right)$ in eq $\mathrm{C} 3$, we obtain

$$
\begin{aligned}
& R_{1}\left(\omega_{1}+\omega_{2}+\omega_{3}, \omega_{1}+\omega_{2}, \omega_{1}\right)=\frac{1}{f\left(\omega_{1}, \omega_{2}, \omega_{3}\right)}(1 \\
& \left(\begin{array}{ll}
\omega_{1}+\omega_{2}+\omega_{3}-\omega_{e^{\prime} g}+\mathrm{i} \frac{k^{\prime}}{2} & -\mathrm{i} \frac{k^{\prime}}{2} \\
-\mathrm{i} \frac{k}{2} & \omega_{1}+\omega_{2}+\omega_{3}-\omega_{e g}+\mathrm{i} \frac{k}{2}
\end{array}\right) \\
& \left(\begin{array}{ll}
\omega_{1}+\omega_{2}+i \frac{k^{\prime}}{2} & -i \frac{k^{\prime}}{2} \\
-\mathrm{i} \frac{k}{2} & \omega_{1}+\omega_{2}+\mathrm{i} \frac{k}{2}
\end{array}\right) \\
& \left(\begin{array}{ll}
\omega_{1}-\omega_{e^{\prime} g}+\mathrm{i} \frac{k^{\prime}}{2} & -\mathrm{i} \frac{k^{\prime}}{2} \\
-\mathrm{i} \frac{k}{2} & \omega_{1}-\omega_{e g}+\mathrm{i} \frac{k}{2}
\end{array}\right)\left(\begin{array}{l}
1-p_{j} \\
p_{j}
\end{array}\right)
\end{aligned}
$$

where

$$
\begin{gathered}
\omega_{e g}=\omega_{e g}, \quad \omega_{e^{\prime} g}=\omega_{e g}+v_{j} \\
\frac{k}{2}=p_{j} K_{j}, \quad \frac{k^{\prime}}{2}=\left(1-p_{j}\right) K_{j} \\
f\left(\omega_{1}, \omega_{2}, \omega_{3}\right)=\left[\left(\omega_{1}+\omega_{2}+\omega_{3}-\omega_{e g}+\mathrm{i} \frac{k}{2}\right) \times\right. \\
\left.\left(\omega_{1}+\omega_{2}+\omega_{3}-\omega_{e^{\prime} g}+\mathrm{i} \frac{k^{\prime}}{2}\right)+\frac{k k^{\prime}}{4}\right] \times \\
{\left[\left(\omega_{1}+\omega_{2}+\mathrm{i} \frac{k}{2}\right)\left(\omega_{1}+\omega_{2}+\mathrm{i} \frac{k^{\prime}}{2}\right)+\frac{k k^{\prime}}{4}\right] \times} \\
{\left[\left(\omega_{1}-\omega_{e g}+\mathrm{i} \frac{k}{2}\right)\left(\omega_{1}-\omega_{e^{\prime} g}+\mathrm{i} \frac{k^{\prime}}{2}\right)+\frac{k k^{\prime}}{4}\right]}
\end{gathered}
$$

or alternatively, in terms of the poles,

$$
\begin{aligned}
& f\left(\omega_{1}, \omega_{2}, \omega_{3}\right)= \\
& \left(\omega_{1}+\omega_{2}+\omega_{3}-\mathrm{i} \Omega_{+}\right)\left(\omega_{1}+\omega_{2}+\omega_{3}-\mathrm{i} \Omega_{-}\right)\left(\omega_{1}+\omega_{2}\right) \times \\
& \left(\omega_{1}+\omega_{2}+\mathrm{i} K\right)\left(\omega_{1}-\mathrm{i} \Omega_{+}\right)\left(\omega_{1}-\mathrm{i} \Omega_{-}\right)(\mathrm{C} 14)
\end{aligned}
$$

with

$$
\begin{gathered}
\Omega_{+}=\Omega_{j}+\frac{b_{j}}{2} \\
\Omega_{-}=-\Omega_{j}+\frac{b_{j}}{2}
\end{gathered}
$$

Similarly, one has

$$
\begin{aligned}
& R_{2}\left(\omega_{1}+\omega_{2}+\omega_{3}, \omega_{1}+\omega_{2}, \omega_{1}\right)=\frac{1}{f^{\prime}\left(\omega_{1}, \omega_{2}, \omega_{3}\right)}\left(\begin{array}{ll}
1 & 1
\end{array}\right) \times \\
& \left(\begin{array}{ll}
\omega_{1}+\omega_{2}+\omega_{3}-\omega_{e^{\prime} g}+\mathrm{i} \frac{k^{\prime}}{2} & -\mathrm{i} \frac{k^{\prime}}{2} \\
-\mathrm{i} \frac{k}{2} & \omega_{1}+\omega_{2}+\omega_{3}-\omega_{e g}+\mathrm{i} \frac{k}{2}
\end{array}\right) \times \\
& \left(\begin{array}{ll}
\omega_{1}+\omega_{2}+\mathrm{i} \frac{k^{\prime}}{2} & -\mathrm{i} \frac{k^{\prime}}{2} \\
-\mathrm{i} \frac{a}{2} k & \omega_{1}+\omega_{2}+\mathrm{i} \frac{k}{2}
\end{array}\right) \times \\
& \left(\begin{array}{ll}
\omega_{1}+\omega_{e^{\prime} g}-\mathrm{i} \frac{k^{\prime}}{2} & \mathrm{i} \frac{k^{\prime}}{2} \\
\mathrm{i} \frac{k}{2} & \omega_{1}+\omega_{e g}-\mathrm{i} \frac{k}{2}
\end{array}\right)\left(\begin{array}{l}
1-p_{j} \\
p_{j}
\end{array}\right)
\end{aligned}
$$

where

$$
\begin{aligned}
& f^{\prime}\left(\omega_{1}, \omega_{2}, \omega_{3}\right)= \\
& \left(\omega_{1}+\omega_{2}+\omega_{3}-\mathrm{i} \Omega_{+}\right)\left(\omega_{1}+\omega_{2}+\omega_{3}-\mathrm{i} \Omega_{-}\right)\left(\omega_{1}+\omega_{2}\right) \times
\end{aligned}
$$$$
\left(\omega_{1}+\omega_{2}+\mathrm{i} K\right)\left(\omega_{1}+\mathrm{i} \Omega_{+}\right)\left(\omega_{1}+\mathrm{i} \Omega_{-}\right)
$$

\section{Appendix D: Third-Order Response in the Time-Domain}

In this Appendix we derive the time-domain third-order response for the TLS model starting from the frequency-domain expression derived in Appendix C. The third-order response function can be written in terms of the Liouville-space Green functions, ${ }^{3}$

$$
\begin{aligned}
& R_{1}\left(t_{3}, t_{2}, t_{1}\right) \equiv\left\langle\left\langle V_{e q}\left|G_{e g}\left(t_{3}\right) V_{e g, e e} G_{e e}\left(t_{2}\right) V_{e e, e g} G_{e g}\left(t_{1}\right) V_{e g, g g}\right| \rho_{g}\right\rangle\right\rangle \\
& R_{2}\left(t_{3}, t_{2}, t_{1}\right) \equiv\left\langle\left\langle V_{e q}\left|G_{e g}\left(t_{3}\right) V_{e g, e e} G_{e e}\left(t_{2}\right) V_{e e, e g} G_{e g}\left(t_{1}\right) V_{g e, g g}\right| \rho_{g}\right\rangle\right\rangle \\
& R_{3}\left(t_{3}, t_{2}, t_{1}\right) \equiv\left\langle\left\langle V_{e q}\left|G_{e g}\left(t_{3}\right) V_{e g, e e} G_{e e}\left(t_{2}\right) V_{g g, g e} G_{g e}\left(t_{1}\right) V_{g e, g g}\right| \rho_{g}\right\rangle\right\rangle \\
& R_{4}\left(t_{3}, t_{2}, t_{1}\right) \equiv\left\langle\left\langle V_{e q}\left|G_{e g}\left(t_{3}\right) V_{e g, g g} G_{g g}\left(t_{2}\right) V_{g g, e g} G_{e g}\left(t_{1}\right) V_{e g, g g}\right| \rho_{g}\right\rangle\right\rangle
\end{aligned}
$$

$R_{1}\left(t_{3}, t_{2}, t_{1}\right)$ can be derived from $R_{1}\left(\omega_{1}+\omega_{2}+\omega_{3}, \omega_{1}+\omega_{2}, \omega_{1}\right)$ via inverse Fourier transforms B10:

$$
\begin{aligned}
& R_{1}\left(t_{3}, t_{2}, t_{1}\right)=\mathrm{e}^{-\mathrm{i}\left(\omega_{e g}+v_{j} / 2\right)\left(t_{1}+t_{3}\right)-\left(K_{j} / 2\right)\left(t_{1}+t_{2}+t_{3}\right)}\left(\begin{array}{ll}
1 & 1
\end{array}\right) \\
& \left(\begin{array}{ll}
\cosh \left(\Omega_{j} t_{3}\right)+g_{j} \sinh \left(\Omega_{j} t_{3}\right) & \frac{k^{\prime}}{2 \Omega_{j}} \sinh \left(\Omega_{j} t_{3}\right) \\
\frac{k}{2 \Omega_{j}} \sinh \left(\Omega_{j} t_{3}\right) & \cosh \left(\Omega_{j} t_{3}\right)-g_{j} \sinh \left(\Omega_{j} t_{3}\right)
\end{array}\right)\left(\begin{array}{ll}
\cosh \left(\frac{K_{j} t_{2}}{2}\right)+\left(1-2 p_{j}\right) \sinh \left(\frac{K_{j} t_{2}}{2}\right) & \frac{k^{\prime}}{K} \sinh \left(\frac{K_{j} t_{2}}{2}\right) \\
\frac{k}{K} \sinh \left(\frac{K_{j} t_{2}}{2}\right) & \cosh \left(\frac{K_{j} t_{2}}{2}\right)-\left(1-2 p_{j}\right) \sinh \left(\frac{K_{j} t_{2}}{2}\right)
\end{array}\right) \times \\
& \left(\begin{array}{ll}
\cosh \left(\Omega_{j} t_{1}\right)+g_{j} \sinh \left(\Omega_{j} t_{1}\right) & \frac{k^{\prime}}{2 \Omega_{j}} \sinh \left(\Omega_{j} t_{1}\right) \\
\frac{k}{2 \Omega_{j}} \sinh \left(\Omega_{j} t_{1}\right) & \cosh \left(\Omega_{j} t_{1}\right)-g_{j} \sinh \left(\Omega_{j} t_{1}\right)
\end{array}\right)\left(\begin{array}{l}
1-p_{j} \\
p_{\mathrm{j}}
\end{array}\right)
\end{aligned}
$$


where we have introduced

$$
g_{j}=\frac{b_{j}}{2 \Omega_{j}}-\frac{p_{j} K_{j}}{\Omega_{j}}
$$

Similarly, $R_{2}\left(t_{3}, t_{2}, t_{1}\right)$ assumes the form

$$
\begin{aligned}
& R_{2}\left(t_{3}, t_{2}, t_{1}\right)=\mathrm{e}^{i\left(\omega_{e g}+v_{j} / 2\right)\left(t_{1}-t_{3}\right)-\left(K_{j} / 2\right)\left(t_{1}+t_{2}+t_{3}\right)}\left(\begin{array}{ll}
1 & 1
\end{array}\right) \times \\
& \left(\begin{array}{ll}
\cosh \left(\Omega_{j} t_{3}\right)+g \sinh \left(\Omega_{j} t_{3}\right) & \frac{k^{\prime}}{2 \Omega_{j}} \sinh \left(\Omega_{j} t_{3}\right) \\
\frac{k}{2 \Omega_{j}} \sinh \left(\Omega_{j} t_{3}\right) & \cosh \left(\Omega_{j} t_{3}\right)-g \sinh \left(\Omega_{j} t_{3}\right)
\end{array}\right)\left(\begin{array}{ll}
\cosh \left(\frac{K_{j} t_{2}}{2}\right)+\left(1-2 p_{j}\right) \sinh \left(\frac{K_{j} t_{2}}{2}\right) & \frac{k^{\prime}}{K} \sinh \left(\frac{K_{j} t_{2}}{2}\right) \\
\frac{k}{K} \sinh \left(\frac{K_{j} t_{2}}{2}\right) & \cosh \left(\frac{K_{j} t_{2}}{2}\right)-\left(1-2 p_{j}\right) \sinh \left(\frac{K_{j} t_{2}}{2}\right)
\end{array}\right) \times \\
& \left(\begin{array}{ll}
\cosh \left(\Omega_{j}^{*} t_{1}\right)+g_{j}^{*} \sinh \left(\Omega_{j}^{*} t_{1}\right) & \frac{k^{\prime}}{2 \Omega_{j}^{*}} \sinh \left(\Omega_{j}^{*} t_{1}\right) \\
\frac{k}{2 \Omega_{j}^{*}} \sinh \left(\Omega_{j}^{*} t_{1}\right) & \cosh \left(\Omega_{j}^{*} t_{1}\right)-g_{j}^{*} \sinh \left(\Omega_{j}^{*} t_{1}\right)
\end{array}\right)\left(\begin{array}{l}
1-p_{j} \\
p_{j}
\end{array}\right) \text { (D7) }
\end{aligned}
$$

where

$$
\begin{gathered}
\Omega_{j}^{*}=\sqrt{\frac{b_{j}^{* 2}}{4}+c_{j}}=\sqrt{\frac{K_{j}^{2}}{4}-\frac{v_{j}^{2}}{4}+\mathrm{i}\left(p_{j}-\frac{1}{2}\right) v_{j} K_{j}} \\
g_{j}^{*}=\frac{b_{j}^{*}}{2 \Omega_{j}^{*}}-\frac{p_{j} K_{j}}{\Omega_{j}^{*}}
\end{gathered}
$$

We further simplify $R_{1}\left(t_{3}, t_{2}, t_{1}\right)$ as

$$
\begin{aligned}
& R_{1}\left(t_{3}, t_{2}, t_{1}\right)=\mathrm{e}^{-\mathrm{i}\left(\omega_{e g}+v_{j} / 2\right)\left(t_{1}+t_{3}\right)-\left(K_{j} / 2\right)\left(t_{1}+t_{2}+t_{3}\right)}\left(F_{j}\left(t_{3}\right), G_{j}\left(t_{3}\right)\right) \times \\
& \left(\begin{array}{ll}
\cosh \left(\frac{K_{j} t_{2}}{2}\right)+\left(1-2 p_{j}\right) \sinh \left(\frac{K_{j} t_{2}}{2}\right) & 2\left(1-p_{j}\right) \sinh \left(\frac{K_{j} t_{2}}{2}\right) \\
2 p_{j} \sinh \left(\frac{K_{j} t_{2}}{2}\right) & \cosh \left(\frac{K_{j} t_{2}}{2}\right)-\left(1-2 p_{j}\right) \sinh \left(\frac{K_{j} t_{2}}{2}\right)
\end{array}\right)\left(\begin{array}{l}
2\left(1-p_{j}\right) \sinh \left(\frac{K_{j} t_{2}}{2}\right) \\
\cosh \left(\frac{K_{j} t_{2}}{2}\right)-\left(1-2 p_{j}\right) \sinh \left(\frac{K_{j} t_{2}}{2}\right)
\end{array}\right)\left(\begin{array}{l}
\left(1-p_{j}\right) F_{j}\left(t_{1}\right) \\
p_{j} G_{j}\left(t_{1}\right)
\end{array}\right)
\end{aligned}
$$

with

$$
\begin{gathered}
b_{j}^{*}=K_{j}-\mathrm{i} v_{j} \\
F_{j}(t)=\cosh \left(\Omega_{j} t\right)+\frac{b_{j}}{2 \Omega_{j}} \sinh \left(\Omega_{j} t\right) \\
G_{j}(t)=\cosh \left(\Omega_{j} t\right)+\frac{b_{j}^{*}}{2 \Omega_{j}} \sinh \left(\Omega_{j} t\right)
\end{gathered}
$$

$R_{2}\left(t_{3}, t_{2}, t_{1}\right)$ is similarly given by

$$
\left.\begin{array}{rl}
R_{2}\left(t_{3}, t_{2}, t_{1}\right)=\mathrm{e}^{\mathrm{i}\left(\omega_{e g}+v_{j} / 2\right)\left(t_{1}-t_{3}\right)-\left(K_{j} / 2\right)\left(t_{1}+t_{2}+t_{3}\right)}\left(F_{j}\left(t_{3}\right), G_{j}\left(t_{3}\right)\right) \times \\
\operatorname{losh}\left(\frac{K_{j} t_{2}}{2}\right)+\left(1-2 p_{j}\right) \sinh \left(\frac{K_{j} t_{2}}{2}\right) & 2\left(1-p_{j}\right) \sinh \left(\frac{K_{j} t_{2}}{2}\right) \\
2 p_{j} \sinh \left(\frac{K_{j} t_{2}}{2}\right) & \cosh \left(\frac{K_{j} t_{2}}{2}\right)-\left(1-2 p_{j}\right) \sinh \left(\frac{K_{j} t_{2}}{2}\right)
\end{array}\right)\left(\begin{array}{l}
\left(1-p_{j}\right) F_{j}^{*}\left(t_{1}\right) \\
p_{j} G_{j}^{*}\left(t_{1}\right)
\end{array}\right)
$$

with

$$
\begin{aligned}
F_{j}^{*}(t) & =\cosh \left(\Omega_{j}^{*} t\right)+\frac{b_{j}^{*}}{2 \Omega_{j}^{*}} \sinh \left(\Omega_{j}^{*} t\right) \\
G_{j}^{*}(t) & =\cosh \left(\Omega_{j}^{*} t\right)+\frac{b_{j}}{2 \Omega_{j}^{*}} \sinh \left(\Omega_{j}^{*} t\right)
\end{aligned}
$$




\section{Appendix E: Fourth-Order Cumulant Expressions for the Nonlinear Response of a Spin Bath}

In this Appendix we carry out the cumulant expansion for the stochastic model of a spin bath. To the fourth order in $v / k$, we obtain

$$
\begin{aligned}
& F_{1}\left(t_{3}, t_{2}, t_{1}\right)=F_{4}\left(t_{3}, t_{2}, t_{1}\right)+\left\langle\left(\frac{v}{k}\right)^{3} \delta\left(t_{3}, t_{2}, t_{1}\right)\right\rangle_{c} \\
& F_{2}\left(t_{3}, t_{2}, t_{1}\right)=F_{3}\left(t_{3}, t_{2}, t_{1}\right)+\left\langle\left(\frac{v}{k}\right)^{3} \delta\left(t_{3}, t_{2}, t_{1}\right)\right\rangle_{c} \\
& F_{3}\left(t_{3}, t_{2}, t_{1}\right)=-\mathrm{i}\left\langle\left(\frac{v}{k}\right)^{3} p(1-p)(1-2 p) k t_{2} \mathrm{e}^{-k t_{2}}\left(1-\mathrm{e}^{-k t_{1}}\right) \times\right. \\
& \left.\left[\left(k t_{2}+2\right)\left(1-\mathrm{e}^{-k t_{3}}\right)-2 k t_{3}\right]\right\rangle_{c}+ \\
& \left\langle( \frac { v } { k } ) ^ { 4 } \frac { p ( 1 - p ) } { 2 } \mathrm { e } ^ { - k t _ { 2 } } \left\{( 1 - 2 p ) ^ { 2 } \left[ k t_{2} h\left(t_{3}, t_{2}, t_{1}\right)+\right.\right.\right. \\
& \left.4 k t_{1}\left(1-\mathrm{e}^{-k t_{3}}\right)+4 k t_{3}\left(1-\mathrm{e}^{-k t_{1}}\right)-4 k^{2} t_{1} t_{3}\right]+ \\
& \left(1-\mathrm{e}^{-k t_{1}}\right)\left(1-\mathrm{e}^{-k t_{3}}\right)\left[k t_{2}+2 p(1-p) \mathrm{e}^{-k t_{2}}\left(1+\mathrm{e}^{-k t_{1}-k t_{3}}\right)-\right. \\
& \left.\left.\left.2 p(1-p)\left(\mathrm{e}^{-k t_{1}}+\mathrm{e}^{-k t_{3}}\right)\right]\right\}\right\rangle_{c} \\
& F_{4}\left(t_{3}, t_{2}, t_{1}\right)=-\mathrm{i}\left\langle\left(\frac{v}{k}\right)^{3} p(1-p)(1-2 p) k t_{2} \mathrm{e}^{-k t_{2}}\left(1-\mathrm{e}^{-k t_{1}}\right) \times\right. \\
& \left.\left(1-\mathrm{e}^{-k t_{3}}\right)\right\rangle_{c}+\left\langle( \frac { v } { k } ) ^ { 4 } \frac { p ( 1 - p ) } { 2 } \mathrm { e } ^ { - k t _ { 2 } } \left\{(1-2 p)^{2} k t_{2} h\left(t_{3}, t_{2}, t_{1}\right)+\right.\right. \\
& \left(1-\mathrm{e}^{-k t_{1}}\right)\left(1-\mathrm{e}^{-k t_{3}}\right)\left[k t_{2}+2 p(1-p)\left(1-\mathrm{e}^{-k t_{2}}\right) \times\right. \\
& \left.\left.\left.\left(\mathrm{e}^{-k t_{1}}+\mathrm{e}^{-k t_{3}}\right)\right]\right\}\right\rangle_{c}
\end{aligned}
$$

where the auxiliary functions $h\left(t_{3}, t_{2}, t_{1}\right)$ and $\delta\left(t_{3}, t_{2}, t_{1}\right)$ are defined as

$$
\begin{array}{r}
h\left(t_{3}, t_{2}, t_{1}\right)=2 k t_{1} \mathrm{e}^{-k t_{1}}\left(1-\mathrm{e}^{-k t_{3}}\right)+2 k t_{3} \mathrm{e}^{-k t_{3}}\left(1-\mathrm{e}^{-k t_{1}}\right)- \\
\left(k t_{2}+5\right)\left(1-\mathrm{e}^{-k t_{1}}\right)\left(1-\mathrm{e}^{-k t_{3}}\right) \\
\delta\left(t_{3}, t_{2}, t_{1}\right)=2 \mathrm{i} p(1-2 p)(1-p)\left[k t_{2}\left(1-\mathrm{e}^{-k t_{3}}\right)+\right. \\
\left.k t_{3}\left(1-\mathrm{e}^{-k t_{2}}\right)-2\left(1-\mathrm{e}^{-k t_{2}}\right)\left(1-\mathrm{e}^{-k t_{3}}\right)\right]
\end{array}
$$

$F_{n}(n=1,2,3,4)$ vanish to second order in $v / k$, pointing to the fact that to second order in $v / k$ the TLS model is equivalent to the Brownian oscillator model. However, third- and fourthorder terms are nonzero. For $F_{1}$ and $F_{4}$, the differences are

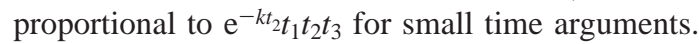

\section{Appendix F: Line-Shape Functions for the Boson and the Spin Bath}

In this Appendix, we give the expressions for the line-shape functions $g^{\mathrm{BO}}(t)$ and $g^{\mathrm{TLS}}(t)$ of the boson and spin bath, respectively. In eq 5.3, we presented the line-shape function for the boson bath $g^{\mathrm{BO}}(t)$ in terms of the spectral density function $C^{\prime \prime}(\omega)$. Alternatively, $g^{\mathrm{BO}}(t)$ can be written as

$$
\begin{gathered}
g^{\mathrm{BO}}(t)=\Delta^{2} \int_{0}^{t} \mathrm{~d} \tau_{2} \int_{0}^{\tau_{2}} \mathrm{~d} \tau_{1} M^{\prime}\left(\tau_{1}\right)-\mathrm{i} \lambda \int_{0}^{t} \mathrm{~d} \tau\left[1-M^{\prime \prime}(\tau)\right] \\
M^{\prime}(t)=\frac{1}{\pi \Delta^{2}} \int_{0}^{\infty} \mathrm{d} \omega C^{\prime \prime}(\omega) \operatorname{coth} \frac{\beta \hbar \omega}{2} \cos (\omega t) \\
M^{\prime \prime}(t)=\frac{1}{\pi \lambda} \int_{0}^{\infty} \mathrm{d} \omega \frac{C^{\prime \prime}(\omega)}{\omega} \cos (\omega t) \\
\Delta^{2}=\frac{1}{\pi} \int_{0}^{\infty} \mathrm{d} \omega C^{\prime \prime}(\omega) \operatorname{coth} \frac{\beta \hbar \omega}{2}(\omega) \\
\lambda=\frac{1}{\pi} \int_{0}^{\infty} \mathrm{d} \omega \frac{C^{\prime \prime}(\omega)}{\omega}
\end{gathered}
$$

The meromorphic funtion $\operatorname{coth}(\beta \hbar \omega / 2)$ may be expanded as a summation over its poles, which are the Matsubara frequencies for bosons: ${ }^{74}$

$$
\operatorname{coth} \frac{\beta \hbar \omega}{2}=\frac{2}{\hbar \beta} \sum_{n} \frac{1}{\mathrm{i} v_{n}-\omega}
$$

with

$$
v_{n}=\frac{2 n \pi}{\hbar \beta}, \quad n=0, \pm 1, \pm 2, \ldots
$$

This allows representing $M^{\prime}(t)$ and $\Delta^{2}$ in terms of the Matsubara frequencies $v_{n}$ by means of contour integrations:

$$
\Delta^{2}=\lambda \Lambda \cot \frac{\hbar \beta \Lambda}{2}+\frac{4 \lambda \Lambda}{\beta} \sum_{n} \frac{v_{n}}{v_{n}^{2}-\Lambda^{2}}
$$

$$
M^{\prime}(t)=\frac{1}{\Delta^{2}}\left(\lambda \Lambda \cot \frac{\hbar \beta \Lambda}{2} \mathrm{e}^{-\Lambda t}+\frac{4 \lambda \Lambda}{\beta} \sum_{n} \frac{v_{n} \mathrm{e}^{-v_{n} t}}{v_{n}^{2}-\Lambda^{2}}\right)
$$

Contour integrations also yield $M^{\prime \prime}(t)$ :

$$
M^{\prime \prime}(t)=\mathrm{e}^{-\Lambda t}
$$

with eq F5 reducing to an identity.

The line-shape function $g(t)=g^{\prime}(t)+\mathrm{i} g^{\prime \prime}(t)$ is therefore obtained from eq F1 as

$$
\begin{array}{r}
g^{\prime}(t)=\frac{\lambda}{\Lambda} \cot \frac{\hbar \beta \Lambda}{2}\left(\mathrm{e}^{-\Lambda t}+\Lambda t-1\right)+\frac{4 \lambda \Lambda}{\hbar \beta} \sum_{n=1}^{\infty} \frac{\mathrm{e}^{-v_{n} t}+v_{n} t-1}{v_{n}\left(v_{n}{ }^{2}-\Lambda^{2}\right)} \\
g^{\prime \prime}(t)=-\frac{\lambda}{\Lambda} \cot \frac{\hbar \beta \Lambda}{2}\left(\mathrm{e}^{-\Lambda t}+\Lambda t-1\right)
\end{array}
$$

The summation in eq F11 can be carried out, and results are expressed in terms of special functions: 


$$
\begin{aligned}
& g^{\prime}(t)=\frac{\lambda}{\Lambda} \cot \frac{\hbar \beta \Lambda}{2}\left(\mathrm{e}^{-\Lambda t}+\Lambda t-1\right)+ \\
& \frac{\lambda}{\pi \Lambda}\left[2 \gamma+2 \log \left(1-\mathrm{e}^{-v t}\right)+\mathrm{e}^{-v t} \Phi\left(\mathrm{e}^{-v t}, 1, \frac{v-\Lambda}{v}\right)+\right. \\
& \mathrm{e}^{-v t} \Phi\left(\mathrm{e}^{-v t}, 1, \frac{v+\Lambda}{v}\right)+(1-\Lambda t) \Psi^{[0]}\left(\frac{v-\Lambda}{v}\right)+ \\
&\left.(1+\Lambda t) \Psi^{[0]}\left(\frac{v+\Lambda}{v}\right)\right]
\end{aligned}
$$

where $v=2 \pi / \hbar \beta, \gamma$ is the Euler's constant $(\approx 0.577216), \Phi$ $(z, s, a)$ is the Lerch transcendent, and $\Psi^{[n]}(z)$ is the Digamma function.

For the spin bath, we have

$$
\begin{gathered}
g^{\mathrm{TLS}}(t)=\Delta^{2} \int_{0}^{t} \mathrm{~d} \tau_{2} \int_{0}^{\tau_{2}} \mathrm{~d} \tau_{1} M^{\prime}\left(\tau_{1}\right)-\mathrm{i} \lambda \int_{0}^{t} \mathrm{~d} \tau\left[1-M^{\prime \prime}(\tau)\right] \\
M^{\prime}(t)=\frac{1}{\pi \Delta^{2}} \int_{0}^{\infty} \mathrm{d} \omega C^{\prime}(\omega) \cos (\omega t) \\
M^{\prime \prime}(t)=\frac{1}{\pi \lambda} \int_{0}^{\infty} \mathrm{d} \omega \frac{C^{\prime}(\omega)}{\omega} \tanh \frac{\beta \hbar \omega}{2} \cos (\omega t) \\
\Delta^{2}=\frac{1}{\pi} \int_{0}^{\infty} \mathrm{d} \omega C^{\prime}(\omega) \\
\lambda=\frac{1}{\pi} \int_{0}^{\infty} \frac{C^{\prime}(\omega)}{\omega} \tanh \frac{\beta \hbar \omega}{2}
\end{gathered}
$$

The line-shape functions for the spin bath can then be obtained as

$$
\begin{gathered}
g^{\prime}(t)=\frac{p(1-p) v^{2}}{\Lambda^{2}}\left(\mathrm{e}^{-\Lambda t}+\Lambda t-1\right) \\
g^{\prime \prime}(t)=-p(1-p) v^{2}\left[\frac{1}{\Lambda^{2}} \tan \frac{\hbar \beta \Lambda}{2}\left(\mathrm{e}^{-\Lambda t}+\Lambda t-1\right)+\right. \\
\left.\frac{4 \Lambda}{\hbar \beta} \sum_{n=0}^{\infty} \frac{\mathrm{e}^{-v_{n} t}+v_{n} t-1}{v_{n}^{2}\left(\Lambda^{2}-v_{n}^{2}\right)}\right]
\end{gathered}
$$

where

$$
v_{n}=\frac{(2 n+1) \pi}{\hbar \beta}
$$

We expand $\tan (\hbar \beta \omega / 2)$ as a summation over its poles, which are the mastubara frequencies for fermions: ${ }^{74}$

$$
\tan \frac{\beta \hbar \omega}{2}=-\frac{2}{\hbar \beta} \sum_{n} \frac{1}{\mathrm{i} v_{n}-\omega}
$$

with

$$
v_{n}=\frac{(2 n+1) \pi}{\hbar \beta}, \quad n=0, \pm 1, \pm 2, \ldots
$$

Performing contour intergrations, one obtains from eqs F15F18

$$
\begin{gathered}
\Delta^{2}=p(1-p) v^{2} \\
M^{\prime}=\mathrm{e}^{-\Lambda t}
\end{gathered}
$$

$$
\lambda=\frac{p(1-p) v^{2}}{\Lambda} \tan \frac{\hbar \beta \Lambda}{2}+\frac{4 \Lambda}{\hbar \beta} \sum_{n} \frac{1}{v_{n}\left(\Lambda^{2}-v_{n}^{2}\right)}
$$

$$
M^{\prime \prime}(t)=\frac{p(1-p) v^{2}}{\Lambda \lambda} \mathrm{e}^{-\Lambda t} \tan \frac{\hbar \beta \Lambda}{2}+\frac{4 \Lambda}{\hbar \beta \lambda} \sum_{n} \frac{\mathrm{e}^{-v_{n} t}}{v_{n}\left(\Lambda^{2}-v_{n}{ }^{2}\right)}
$$

\section{Appendix G: Limiting Cases of the Linear Response}

In Section IV, we have examined the limit of weak chromophore-TLS coupling. Here we look into the opposite limit of slow TLS modulation of the chromophore transition frequency. The static limit $(K=0)$ can be seen as the case of defect-induced inhomogeneous broadening. ${ }^{62,75}$

1. Slow Modulation. In the opposite limit $v \gg k$, we expand $g_{c}(t)$ to the second order in $k / v$ :

$$
\begin{aligned}
& \frac{1}{N} g_{c}(t)=-\left\langle\ln \left[1-p\left(1-\mathrm{e}^{-\mathrm{i} v t}\right)\right]\right\rangle_{c}+ \\
& \left|\frac{k}{v} 2 p(1-p) \frac{v t \cos \frac{v t}{2}-2 \sin \frac{v t}{2}}{\cos \frac{v t}{2}+\mathrm{i}(1-2 p) \sin \frac{v t}{2}}\right|_{c}+ \\
& \left\langle\frac{k^{2}}{v^{2}\left[\cos \frac{v t}{2}+\mathrm{i}(1-2 p) \sin \frac{v t}{2}\right]^{2}}[(3-8 p(1-p))\right. \\
& (1-\cos v t)+\mathrm{i} v t(1-2 p)(2+\cos v t)- \\
& \left.\left.\frac{1}{2} v^{2} t^{2}(2 p-1)^{2}-\left(2 p^{2}+(1-2 p)(3 \mathrm{i}+v t)\right) \sin \right]\right)
\end{aligned}
$$

In the high-temperature limit $\kappa \ll 1, g_{c}(t)$ reduces to

$$
\begin{aligned}
& \frac{1}{N} g_{c}(t)=-\left\langle\ln \frac{1+\mathrm{e}^{-\mathrm{i} v t}}{2}\right\rangle_{c}+\left\langle\frac{k}{v}\left(\frac{v t}{2}-\tan \frac{v t}{2}\right)\right\rangle_{c}+ \\
& \left\langle\frac{k^{2} 1-\cos t v-t v \sin t v}{v^{2}}\right\rangle_{c}
\end{aligned}
$$

1a. Static Limit $k=0$. In this limit, $p$ is actually the occupation probability of the excited TLS, which is equivalant to the defect density in the lattice model of inhomogeneous line shapes. ${ }^{62,75,76}$

Upon expanding the first term of eq $\mathrm{G} 2$ to ninth order in $v t$, one obtains 


$$
\begin{gathered}
g(t)=\sum_{j} p\left(-\mathrm{i} v_{j} t\right)+\sum_{j}\left[\frac{x}{2 !}\left(-\mathrm{i} v_{j} t\right)^{2}+\frac{(1-2 p) x}{3 !}\left(-\mathrm{i} v_{j} t\right)^{3}+\right. \\
\frac{1}{4 !}\left(x-6 x^{2}\right)\left(-\mathrm{i} v_{j} t\right)^{4}+\frac{1-2 p}{5 !}\left(x-12 x^{2}\right)\left(-\mathrm{i} v_{j} t\right)^{5}+ \\
\frac{1}{6 !}\left(x-30 x^{2}+120 x^{3}\right)\left(-\mathrm{i} v_{j} t\right)^{6}+ \\
\frac{1-2 p}{7 !}\left(x-60 x^{2}+360 x^{3}\right)\left(-\mathrm{i} v_{j} t\right)^{7}+ \\
\frac{1}{8 !}\left(x-126 x^{2}+1680 x^{3}-5040 x^{4}\right)\left(-\mathrm{i} v_{j} t\right)^{8}+ \\
\left.\frac{1-2 p}{9 !}\left(x-252 x^{2}+5040 x^{3}-20160 x^{4}\right)\left(-\mathrm{i} v_{j} t\right)^{9}\right](\mathrm{G} 3)
\end{gathered}
$$

where

$$
x \equiv p(1-p)
$$

Odd-order terms have a factor $1-2 p$ which vanishes at high temperatures, and all terms are proportional to $p(1-p)$. We note that the coefficients of $(v t)^{2 n}$ and $(v t)^{2 n+1}$ contain $n$ terms up to $x^{n}$. It is interesting to observe that the coefficients of $x^{i}$ in odd-order terms $(v t)^{2 n+1}$ is $i$ times those of $x^{i}$ in even-order terms $(v t)^{2 n}$.
At high temperatures $(\kappa \ll 1)$, half of a lattice is occupied by defects. One obtains from eq G3 a Gaussian lineshape, i.e., for $K_{j} \rightarrow 0$ and $T \rightarrow \infty(\kappa \ll 1)$,

$$
g(t)=\sum_{j} \ln \frac{1+\mathrm{e}^{-\mathrm{i} v_{j} t}}{2} \approx-\mathrm{i}-\sum_{2} \sum_{j} v_{j} t-\frac{1}{8} \sum_{j} v_{j}^{2} t^{2}
$$

provided that the higher-order terms

$$
-\sum_{j}\left[\frac{1}{8 \times 4 !}\left(v_{j} t\right)^{4}+\frac{1}{4 \times 6 !}\left(v_{j} t\right)^{6}+\frac{17}{4} \frac{1}{8 !}\left(v_{j} t\right)^{8}+\ldots\right]
$$

are vanishingly small. In order for the above summation to remain finite in the case of dipolar coupling, however, one has to impose a lower cutoff for the chromophore-TLS separation, $a$.

At low temperatures, $p \rightarrow 0$, and most TLS remain in their ground states. One obtains from eq G1

$$
g(t)=\sum_{j} p_{j}\left(1-\mathrm{e}^{\mathrm{i} v_{j} t}\right)
$$

For the dipolar coupling, a Lorentzian line shape emerges as the lower cutoff for the chromophore-TLS separation, $a$, tends to zero. This is discussed in Section IV.

2. High Temperature $\kappa \ll 1$. Performing a high-temperature expansion of eq 4.1 to second order in $(p-1 / 2)$, we obtain

$$
\begin{aligned}
& \frac{1}{N^{2}} g_{c}(t)=-\left\langle-\frac{1}{2}(k+\mathrm{i} v) t\right\rangle_{c}-\left\langle\ln \left[\cosh \left(\frac{1}{2} \sqrt{k^{2}-v^{2} t}\right)+\frac{k}{\sqrt{k^{2}-v^{2}}} \sinh \left(\sqrt{k^{2}-v^{2} t}\right)\right]\right\rangle_{c}+
\end{aligned}
$$

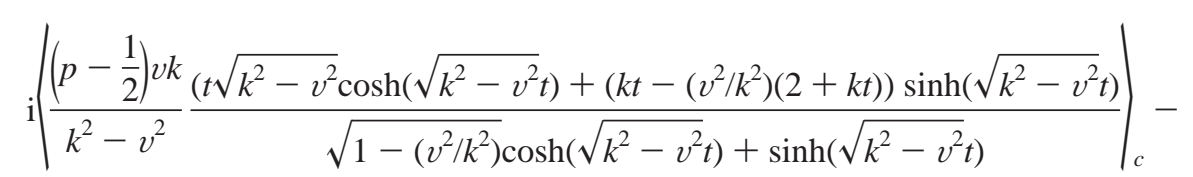

$$
\begin{aligned}
& \left\langle\frac { 1 } { 2 } ( p - \frac { 1 } { 2 } ) ^ { 2 } ( \frac { v k } { k ^ { 2 } - v ^ { 2 } } ) ^ { 2 } \frac { 1 } { [ \sqrt { 1 - ( v ^ { 2 } / k ^ { 2 } } ) \operatorname { c o s h } ( \sqrt { k ^ { 2 } - v ^ { 2 } } t ) + \operatorname { s i n h } ( \sqrt { k ^ { 2 } - v ^ { 2 } } t ) ] ^ { 2 } } \left\{\frac{v^{2}}{k^{2}}\left(1-\frac{v^{2}}{k^{2}}\right)\left(k^{2} t^{2}+4 k t\right)+4-2\left(1-\frac{v^{2}}{k^{2}}\right)^{2}+\right.\right. \\
& \left.\left.2\left[-2+\left(1-\frac{v^{2}}{k^{2}}\right)^{2}+\left(1-\frac{v^{2}}{k^{2}}\right) k t\right] \cosh \left(\sqrt{k^{2}-v^{2}} t\right)+\sqrt{1-\frac{v^{2}}{k^{2}}}\left[k t\left(2-\frac{v^{2}}{k^{2}}\right)+4\left(1-\frac{v^{2}}{k^{2}}\right)-6\right] \sinh \left(\sqrt{k^{2}-v^{2}} t\right)\right\}\right)_{c}
\end{aligned}
$$

\section{Appendix H: Microscopic Model for a Spin Bath}

In this Appendix we introduce a microscopic model of the spin bath and derive a closed expression for the line-shape function. We assume that the chromophore is coupled to a bath represented by a collection of the TLS. The Hamiltonian has the form

$$
\hat{H}=\frac{\Omega}{2} \sigma_{0}^{z}+\sum_{j} \frac{\omega_{j}}{2} \sigma_{j}^{z}+\sum_{j} u_{j} \sigma_{j}^{x} \frac{1+\sigma_{0}^{z}}{2}
$$

or equivalently,

$$
\hat{H}=|g\rangle \hat{H}_{g}\langle g|+| e\rangle\left(\omega_{e g}+\hat{H}_{e}\right)\langle e|
$$

where

$$
\begin{gathered}
\hat{H}_{g}=\sum_{j} \hat{H}_{j}^{g}=\sum_{j} \frac{\omega_{j}}{2} \sigma_{j}^{z} \\
\hat{H}_{e}=\sum_{j} \hat{H}_{j}^{g}+\hat{U}=\sum_{j} \frac{\omega_{j}}{2} \sigma_{j}^{z}+\sum_{j} u_{j} \sigma_{j}^{x}
\end{gathered}
$$

Here $\hat{H}_{j}^{g}$ anf $\hat{H}_{j}^{e}$ are the Hamiltonian of the $j$ th bath spin when the chromophore is in the ground and excited states, respectively, and $\hat{U}$ is the collective bath coordinate. In eq $\mathrm{H} 1 \sigma_{0}^{\alpha}$ represent Pauli matrices of the chromophore, whereas $\sigma_{j}^{\alpha}$ are the Pauli matrices that represent spins of the bath. $\omega_{e g}$ is chromophore frequency; $\omega_{j}$ is the frequency of the $j$ th bath spin, which is decoupled from the chromophore in the ground state of the chromophore but coupled with strength $u_{j}$ in the chromophore 
excited state. For the linear response function $J_{j}(t)$ we have

$$
J_{j}(t)=\operatorname{Tr}\left[\exp \left(-\mathrm{i} H_{j}^{g} t\right) \exp \left(-\beta H_{j}^{g}\right) \exp \left(\mathrm{i} H_{j}^{e} t\right)\right]
$$

where $\exp \left(-\beta H_{j}^{g}\right)$ with $\beta=1 / k_{\mathrm{B}} T$ is the equilibrium density matrix of the $j$ th bath spin. A straightforward calculation yields

$$
\begin{aligned}
J_{j}(t)=\cos \lambda_{j} t \cos \frac{\omega_{j} t}{2}+\frac{\omega_{j}}{2 \lambda_{j}} \sin \lambda_{j} t \sin \frac{\omega_{j} t}{2}+ \\
\quad i \tanh \frac{\beta t}{2}\left[\cos \lambda_{j} t \sin \frac{\omega_{j} t}{2}-\frac{\omega_{j}}{2 \lambda_{j}} \sin \lambda_{j} t \cos \frac{\omega_{j} t}{2}\right]
\end{aligned}
$$

where

$$
\lambda_{j}=\sqrt{\left(\frac{\omega_{j}}{2}\right)^{2}+u_{j}^{2}}
$$

Introducing $J(t ; \omega, u)$ with $\omega, u$ substituted for $\omega_{j}, u_{j}$ and the distribution $n(\omega, u)$, we can recast eq $\mathrm{H} 5$ in a form

$$
g(t)=-\int_{0}^{\infty} \frac{\mathrm{d} \omega}{2 \pi} \int_{0}^{\infty} \mathrm{d} u n(\omega, u) \ln \left[F_{j}(t ; \omega, u)\right]
$$

where we use the normalization

$$
\int_{0}^{\infty} \frac{\mathrm{d} \omega}{2 \pi} \int_{0}^{\infty} \mathrm{d} u n(\omega, u)=1
$$

Equations $\mathrm{H} 8$ and H6 express the line-shape function $g(t)$ in terms of the distribution function $n(\omega, u)$ in the general case.

Now we consider the weak coupling limit when $n(\omega, u)$ is nonzero only in the regime $u \ll \omega$. Expanding $\ln \left[F_{j}(t ; \omega, u)\right]$ in powers of $u / \omega$ and retaining terms up to sixth order yields

$$
\begin{gathered}
g(t)= \\
\int_{0}^{\infty} \frac{\mathrm{d} \omega \bar{C}^{(2)}(\omega)}{2 \pi \omega^{2}}\left[(1-\cos \omega t)+\mathrm{i} \tanh \frac{\omega}{2 k_{B} T}(\omega t-\sin \omega t)\right]- \\
\int_{0}^{\infty} \frac{\mathrm{d} \omega \bar{C}^{(4)}(\omega)}{2 \pi} \omega^{4}[(1-\cos \omega t)+\cosh \beta t+\cosh (\mathrm{i} \omega t+\beta t)- \\
\left.\frac{\mathrm{i}}{2} \sin \omega t \sinh (\mathrm{i} \omega t+\beta t)+\mathrm{i} \omega t\left(\sinh (\mathrm{i} \omega t+\beta t)+\frac{1}{2} \sinh \beta t\right)\right]- \\
\int_{0}^{\infty} \frac{\mathrm{d} \omega \quad \bar{C}^{(6)}(\omega)}{2 \pi} \frac{12 \omega^{6} \cosh ^{3} \frac{\beta t}{2}}{2}\{q(t)-2 \mathrm{i} \omega t s(t)+ \\
\left.12 \omega^{2} t^{2}\left[\cosh \frac{\beta t}{2}-\sinh ^{\frac{\beta t}{2}} \sinh (\mathrm{i} \omega t+\beta t)\right]+4 \mathrm{i} \omega^{3} t^{3} \sinh \frac{\beta t}{2}\right\}
\end{gathered}
$$

where

$$
\begin{array}{r}
q(t)=-54+15 \cosh \left(\frac{\beta t}{2}-\mathrm{i} \omega t\right)+33 \cosh \left(\frac{\beta t}{2}+\mathrm{i} \omega t\right)+ \\
6 \cosh \left(\frac{\beta t}{2}+2 \mathrm{i} \omega t\right)-22 \cosh \frac{3 \beta t}{2}+15 \cosh \left(\frac{3 \beta t}{2}+\mathrm{i} \omega t\right)+ \\
6 \cosh \left(\frac{3 \beta t}{2}+2 \mathrm{i} \omega t\right)+\cosh \left(\frac{3 \beta t}{2}+3 \mathrm{i} \omega t\right) \\
s(t)=6 \sinh \frac{\beta t}{2}+3 \sinh \left(\frac{3 \beta t}{2}+\mathrm{i} \omega t\right)+3 \sinh \frac{3 \beta t}{2}+ \\
\left.3 \sinh \left(\frac{3 \beta t}{2}+\mathrm{i} \omega t\right)+\sinh \left(\frac{3 \beta t}{2}+2 \mathrm{i} \omega t\right)\right](\mathrm{H} 11)
\end{array}
$$

Introducing the spectral density $C(\omega)$ by

$$
C^{(2)}(\omega) \equiv \bar{C}^{(2)}(\omega) \tanh \frac{\omega}{2 k_{B} T}
$$

we reproduce the result of the Brownian oscillator model to second order in $u / \omega$ :

$$
\bar{C}^{(2)}(\omega)=\int_{0}^{\infty} \mathrm{d} u u^{2} n(\omega, u)
$$

\section{References and Notes}

(1) Barbara, P. F., Knox, W. H., Mourou, G. A., Zewail, A. H., (Eds.) Ultrafast Phenomena IX; Proceedings of the 9th International Conference; (Springer-Verlag: Berlin, Heidelberg, 1994).

(2) Chergui, M., (Ed.) Femtochemistry: Ultrafast Chemical and Physical Processes in Molecular Systems; Proceedings of The Lausanne Conference; (World Scientific: Singapore, 1996).

(3) Mukamel, S. Principles of Nonlinear Optical Spectroscopy; (Oxford: New York, 1995).

(4) Moerner, W. E., Kador, L. Phys. Rev. Lett. 1989, 65, 2535.

(5) Basché, Th.; Ambrose, W. P.; Werner, W. E. J. Opt. Soc. Am. 1992, $B 9,829$.

(6) Orrit, M.; Bernard, J.; Zumbusch, A.; Personov, R. I. Chem. Phys. Lett. 1992, 196, 595; 1992, 199, 408.

(7) Kettner, R.; Tittel, J.; Basché, Th.; Bräuchle, C. J. Phys. Chem. 1994, 98,6671 .

(8) Fleury, L.; Zumbusch, A.; Orrit, M.; Brown, R.; Bernard, J. J. Lumin $1993,56,15$.

(9) Ambrose, W. P.; Moerner, W. E. Nature 1991, 349, 225. Ambrose, W. P.; Basché, Th.; Moerner, W. E. J. Chem. Phys. 1991, 95, 7150.

(10) Basché, Th.; Moerner, W. E. Nature 1992, 355, 335.

(11) Basché, T.; Moerner, W.; Orrit, M.; E.U.P. Wild, Single-Molecule Optical Detection, Imaging and Spectroscopy; (VCH: Weinheim, 1996).

(12) Zeller, R. C.; Pohl, R. O. Phys. Rev. B 1971, 4, 202.

(13) Anderson, P. W.; Halperin, B. I.; Varma, C. M. Phil. Mag. 1972, $25,1$.

(14) W. A. Phillips, J. Low Temp. Phys. 1972, 7, 35.

(15) Yu, C. C.; Leggett, A. J. Comments Condens. Matter Phys. 1988, $14,23$.

(16) Leggett, A. J. Physica B 1991, 169, 32.

(17) Black, J. L.; Halperin, B. I. Phys. Rev. B 1977, 16, 287.

(18) Heuer, A.; Silbey, R. J. Phys. Rev. B 1994, 49, 144; 1993, 48, 9411; Phys. Rev. Lett. 1993, 70, 3911.

(19) Jankowiak, R.; Small, G. J. Phys. Rev. B 1987, 37, 840; Jankowiak, R.; Hayes, J. M.; Small, G. J. Phys. Rev. B 1988, 38, 2084.

(20) Zumbusch, A.; Fleury, L.; Brown, R.; Bernard, J.; Orrit, M. Phys. Rev. Lett. 1993, 70, 358

(21) Phillips, W. A. Rep. Prog. Phys. 1987, 50, 165.

(22) Joffrin, J.; Levelut, A. J. Phys. (Paris) 1972, 36, 81.

(23) Better, J. F.; Meissner, M. Z. Phys. B 1988, 70, 6.

(24) Holstein, T.; Lyo, S. K.; Orbach, R. In Laser Spectroscopy of Solids; Yen, W. M., Selzen, P. M., Eds.; Springer: Berlin, 1981.

(25) Skinner, J. L.; Trommsdorff, H. P. J. Chem. Phys. 1988, 89, 89

(26) Karpov, V. G.; Klinger, M. 1.; Ignatiev, F. N. Zh. Eksp. Teor. Fiz.

1983, 84, 760 [Sov. Phys. JETP 1983, 57, 439].

(27) Heuer, A.; Silbey, R. J. Phys. Rev. B 1996, 53, 60.

(28) Kelham, S.; Rosenberg, H. M. J. Phys. C 1981, 14, 173.

(29) Graebner, J. E.; Golding, B.; Allen, L. C. Phys. Rev. B 1991, 34, 569.

(30) Buchenau, U.; Galperin, Yu. M.; Gurevich, V. L.; Schober, H. R. Phys. Rev. B 1991, 43, 503.

(31) Anderson, P. W.; Weiss, P. R. Rev. Mod. Phys. 1953, 25, 26.

(32) Anderson, P. W. J. Phys. Soc. Jpn. 1954, 9, 316.

(33) Kubo, K. J. Phys. Soc. Jpn. 1954, 9, 93.

(34) Kubo, R. Adv. Chem. Phys. 1969, 15,101

(35) Kubo, R. In Fluctuation, Relaxation, and Resonance in Magnetic Systems; TerHaar, D., Ed.; Oliver and Boyd: Edinburgh, 1962.

(36) Mims, W. B. Phys. Rev. 1968, 168, 37.

(37) Hu, P.; Hartmann, S. R. Phys. Rev. B 1973, 9, 1.

(38) Hu, P.; Walker, L. R. Phys. Rev. B 1978, 18, 130.

(39) Huber, D. L. J. Lum. 1987, 36, 30.

(40) Suárez, A.; Silbey, R. Chem. Phys. Lett. 1993, 218, 44

(41) Putikka, W. O.; Huber, D. L. Phys. Rev. B 1987, 36, 343.

(42) Maruyama, K.; Shibata, F. Physica A 1988, 149, 44.

(43) Jankowiak, R.; Shu, L.; Kenney, M. J.; Small, G. J. J. Lumin. 1987, $36,29$.

(44) Jankowiak, R.; Small, G. J. Phys. Rev. B 1993, 47, 14805; Chem. Phys. Lett. 1993, 207, 43.

(45) Tanimura, Y.; Takano, H.; Klafter, J. J. Chem. Phys. 1998, 108, 185.

(46) Orrit, M.; Bernard, J. Phys. Rev. Lett. 1990, 65, 2716.

(47) Moerner, W. E. Science 1994, 265, 4. 
(48) Peter Lu, H.; Sunney Xie, X. Nature 1997, 385, 143. Sunney Xie, X. Acc. Chem. Res. 1996, 29, 598. Bian, R. X. et al. Phys. Rev. Lett. 1995, $75,477$.

(49) Tittel, J.; Kettner, R.; Basché, Th.; Bräuchle, C.; Quante, H.; Müllen, K. J. Lumin. 1995, 64, 1.

(50) Kozankiewicz, B.; Bernard, J.; Orrit, M. J. Chem. Phys. 1994, 101, 937.

(51) Reilly, P. D.; Skinner, J. L. J. Chem. Phys. 1994, 101, 95; 1995, 102, 1540; Phys. Rev. Lett. 1993, 71, 425.

(52) Nagasawa, Y.; Passino, S. A.; Fleming, G. R. J. Chem. Phys. 1997, 106, 4840; submitted to J. Chem. Phys.

(53) Passino, S. A.; Nagasawa, Y.; Fleming, G. R. J. Chem. Phys. 1997, 107, 109.

(54) Bardeen, C. J.; Cerullo, G.; Shank, C. V. Chem. Phys. Lett., in press.

(55) Chernyak, V.; Mukamel, S. J. Chem. Phys. 1996, 105, 456.

(56) In early treatments, ${ }^{3} \gamma(\omega)$ was assumed to be independent of frequency, and the self-energy was neglected.

(57) Klauder, J. R.; Anderson, P. W. Phys. Rev. 1961, 125, 91.

(58) Molmer, K.; Castin, Y.; Dalibard, J. J. Opt. Soc. Am. B 1993, 10, 527; Stenholm, S. Quantum Semiclass. Opt. 1996, 8, 297; Webster, F. A.;

Rossky, P. J.; Friesner, R. A. Comput. Phys. Commun. 1991, 63, 494.

(59) Suárez, A.; Silbey, R. J. Phys. Chem. 1994, 98, 732.

(60) Kassner, K.; Silbey, R. J. Phys. Condens. Mater. 1989, 1, 459.
(61) Feller, W. An Introduction to Probability Theory and its Applications, 2nd ed.; (Wiley: New York, 1957); Vol. 1.

(62) Stoneham, A. M. Rev. Mod. Phys. 1969, 41, 8.

(63) Wang, M. C.; Uhlenbeck, G. E. Rev. Mod. Phys. 1945, 17, 32; reprinted in Selected Papers on Noise and Stochastic Processes; Wax, N. Ed.; Dover: New York, 1954).

(64) Mandelbrot, B. B. The Fractal Geometry of Nature; (Freeman: San Francisco, 1982)

(65) Weiss, U. Quantum Disspative Systems; (World Scientific: Singapore, 1993).

(66) Holstein, T.; Primakoff, H. Phys. Rev. B 1940, 58, 109.

(67) Chang, L.-D.; Chakravarty, S. Phys. Rev. B 1985, 31, 15.

(68) Kondo, J. Prog. Theor. Phys. 1964, 32, 3.

(69) Leggett, A. J. et al. Rev. Mod. Phys. 59, 1 (1987).

(70) Schotte, K. P. Z. Phys. 1970, 230, 9.

(71) Zumofen, G.; Klafter, J. Chem. Phys. Lett. 1994, 219, 30.

(72) Zhao, Y.; Bertram, H. N. J. Mag. Mag. Mat. 1992, 114, 32.

(73) Zürcher, U. J. Chem. Phys. 1994, 103, 482.

(74) Mahan, G. D. Many-Particle Physics; (Plenum Press: New York, 1981)

(75) Davies, G. J. Phys. D: Appl. Phys. 1971, 4, 134.

(76) Orth, D. L.; Mashl, R. J.; Skinner, J. L. J. Phys.: Condens. Matter 1993, 5, 2533. 